\title{
Mixed convection in four-sided lid-driven sinusoidally heated porous cavity using stream function-vorticity formulation
}

\author{
Shobha Bagai ${ }^{1} \cdot$ Manoj Kumar $^{2}$ (I) $\cdot{\text { Arvind } \text { Patel }^{2}}^{2}$
}

Received: 15 April 2020 / Accepted: 31 October 2020 / Published online: 24 November 2020

(c) Springer Nature Switzerland AG 2020

\begin{abstract}
This study presents the mixed convection inside a four-sided lid-driven square porous cavity whose right wall is maintained at a sinusoidal temperature condition, the left wall of the cavity is maintained at a cold temperature, while the top and the bottom walls are adiabatic. We have discussed two different cases depending upon the direction of the moving walls. Brinkmann-extended Darcy model is represented in terms of $\psi$ and $\xi$ using the stream function-vorticity formulation to simulate the momentum transfer in the porous medium. This formulation is used to solve the governing equations as a coupled system of equations which consists of the field variables, vorticity $(\xi)$, stream function $(\psi)$, and temperature $(T)$. The velocity components $(u, v)$ are derived from the stream function $(\psi)$ whereas the average Nusselt number is derived from temperature. The stability and consistency of the applied numerical scheme to the considered problem has been proven by matrix method. The numerical results are investigated by ranging the various dimensionless numbers such as Grashof number $\left(10^{3} \leq \mathrm{Gr} \leq 10^{5}\right)$, Darcy number $\left(10^{-1} \leq \mathrm{Da} \leq 10^{-5}\right)$, Reynolds number $(10 \leq \operatorname{Re} \leq 1000)$ and keeping the Prandtl number $(\operatorname{Pr}=0.7)$ fixed.
\end{abstract}

Keywords Mixed convection · Four-sided lid-driven flow · Porous media · Finite difference method · Alternatingdirection-implicit (ADI) method

Mathematics Subject Classification $35 \mathrm{Q} 79 \cdot 76 \mathrm{~S} 05 \cdot 80 \mathrm{~A} 20 \cdot 76 \mathrm{M} 20$

\section{List of symbols}

Greek

$\rho(E)$

$\alpha$

$\beta$

$v$

$\psi$

$\rho$

$\xi$
Spectral radius of $E$, which is the maximum of the moduli of its eigenvalues $\lambda_{i}, i=1,2, \ldots, n$ Thermal diffusivity Coefficient of thermal expansion Kinematic viscosity Dimensionless stream function Density of the fluid Dimensionless vorticity

$\begin{array}{ll}\text { Roman } & \\ \Delta x, \Delta y & \text { Grid spacing along } x \text { and } y \text { axis } \\ \frac{\Delta t}{N u_{l}} & \text { Step length along } t \text {-direction } \\ D a & \text { Average Nusselt number at left wall } \\ g & \text { Darcy number } \\ G r & \text { Acceleration due to gravity } \\ k & \text { Thermol number } \\ L & \text { Length of the cavity } \\ n & \text { Number of grid points } \\ p & \text { Dimensionless pressure } \\ p^{\prime} & \text { Dimensional pressure } \\ \operatorname{Pr} & \text { Prandtl number }\end{array}$

Manoj Kumar, manojransiwal@gmail.com; Shobha Bagai, shobhabagai@gmail.com; Arvind Patel, arvindpatelmath09@gmail.com | ${ }^{1}$ Cluster Innovation Centre, University of Delhi, 3rd Floor University Stadium, G C Narang Road, Delhi 110007 , India. ${ }^{2}$ Department of Mathematics, Faculty of Mathematical Sciences, University of Delhi, Delhi 110007, India. 


$\begin{array}{ll}R e & \text { Reynolds number } \\ T & \text { Dimensionless temperature } \\ t & \text { Dimensionless time } \\ T^{\prime} & \text { Dimensional temperature } \\ t^{\prime} & \text { Dimensional time } \\ T_{c} & \text { Reference temperature } \\ u, v & \text { Dimensionless component of velocity } \\ u^{\prime}, v^{\prime} & \text { Dimensional component of velocity } \\ V_{0} & \text { Reference velocity } \\ x, y & \text { Dimensionless Cartesian co-ordinates } \\ x^{\prime}, y^{\prime} & \text { Dimensional Cartesian co-ordinates }\end{array}$

\section{Subscripts and superscripts}

$\begin{array}{ll}i, j & \text { Grid points } \\ l & \text { Left wall } \\ t & \text { Time level }\end{array}$

\section{Introduction}

The problem of mixed convection and heat transfer inside a four-sided lid-driven square porous cavity has been the subject of exhaustive research during the current years. This is due to its well-known applications [1] in the field of engineering and industries such as heating and cooling of buildings, cooling of electronic devices, boiler tubes, heat exchangers, heating of electric irons, quenching of ingots, furnaces, freezing of foods, cooling of cylinder heads in I.C. engines, etc. Numerical investigation of natural or mixed convection with various heat conditions inside a one, two or four-sided lid-driven cavity with or without hydro-dynamic effect has been conducted by several researchers during the past decades. For the two-sided lid-driven cavity problem, the authors generally put the horizontal or vertical walls in motion, in parallel or antiparallel direction with a uniform speed. In addition to the previous cases, the authors have also examined the case of adjacent moving sidewalls in which they kept the left wall in motion in the downward direction while the top wall moves from the left to right. The temperature boundary conditions of these types of problem are almost similar in all the research conducted by the authors. They generally put the vertical or horizontal walls as adiabatic, while the third wall is hot and the fourth wall is kept at a cold temperature. The four-sided lid-driven cavity problem has also been studied by many researchers during recent years. In four-sided lid-driven cavity problems, the authors generally keep the top wall in motion from left to right, the bottom wall in motion from right to left, the right wall in upwards motion, and the left wall in a downward direction with equal speed. The researchers have used various numerical techniques such as finite difference, finite volume method, a compact fourth-order accurate central difference scheme, FAS multigrid method based on a SIMPLE algorithm, etc. to analyze the fluid flow inside the two or four-sided lid-driven cavity problems.

Vusala and Kumar [1] have numerically investigated the fluid flow in a two-sided lid-driven square cavity whose top and bottom walls are kept at a mixed temperature boundary conditions. They have used the streamfunctionvorticity formulation along with the finite difference ADI method to investigate the heat transfer inside the cavity using the local Nusselt number, for different Prandtl numbers 0.7 (for air) and 6.75 (for water) at low Reynolds numbers. Vusala and Kushawaha [2] have numerically simulated the 2-D unsteady, incompressible flow in a four-sided lid-driven rectangular cavity. They have obtained the numerical solutions for the flow variables as well as local and average Nusselt numbers (to analyze the heat transfer inside the cavity) using the QUICK scheme of finite volume methods. Azwadi et al. [3] have used the Adams-Bashforth method to investigate the fluid flow inside a four-sided lid-driven square cavity, at below the critical Reynolds numbers ( $\mathrm{Re} \leq 127)$. Bagai et al. [4] have examined the problem of heat and mass transfer of a 2-D unsteady incompressible fluid flow in a four-sided lid-driven square cavity with the help of stream functionvorticity formulation and ADI method. Chattopadhyay et al. [6] have investigated the 2-D mixed convection flow in a two-sided lid-driven square cavity for three different cases according to the motion of the vertical walls, with retaining the right wall at a sinusoidal temperature condition and the left wall as cold temperature. Chen et al. [7] have used Keller's continuation method to analyze the multiple solutions and flow bifurcation at various Reynolds numbers inside a four-sided lid-driven square cavity. Karaminejad et al. [8] has studied the two-sided adjacent moving walls and four-sided lid-driven cavity problems using the finite-volume method to analyzes the effect of Reynolds numbers. They have found the symmetric solution at low Reynolds numbers, which become asymmetric with an increase of Reynold numbers in a four-sided driven cavity. Khanafer and Chamkha [9] have used the finite volume method along with the ADI scheme to examine the mixed convection inside a one-sided lid-driven cavity, which contains fluid-saturated porous medium in the occupancy of internal heat generation. Kumar et al. [10] has implemented a time-efficient FAS multigrid method along with the SIMPLE algorithm of finite volume method to examine the multiple stable solutions of two-sided adjacent and four-sided lid-driven cavity problem. Munshi et al. [11] has explored the lid-driven porous square cavity whose sides walls are linearly heated and contain an elliptic shape adiabatic block in the middle of the cavity, using the finite element Galerkin method of weighted residuals. Saha et al. [13] have adopted Galerkin weighted 


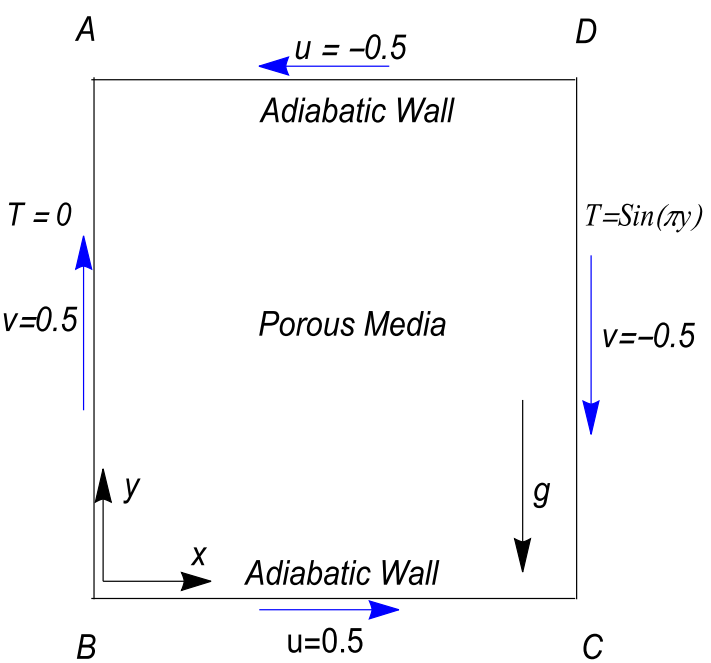

(a) Case 1

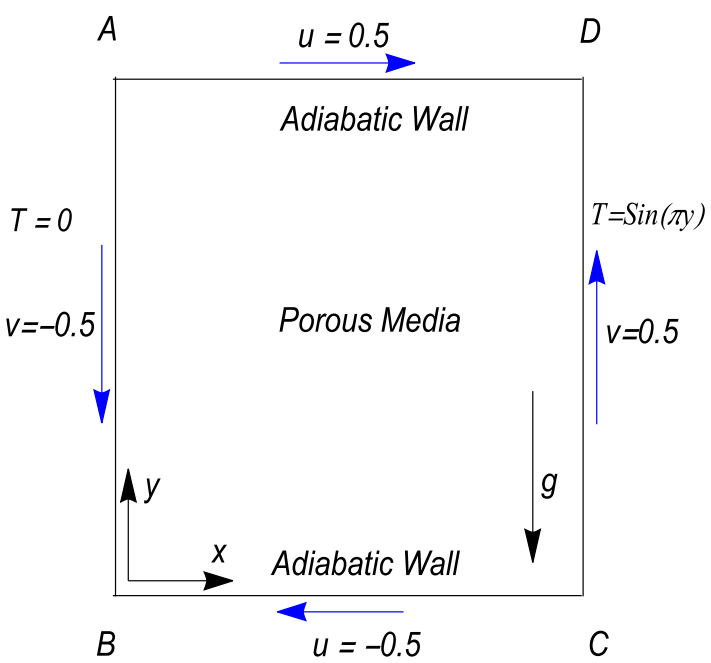

(b) Case 2

Fig. 1 Coordinate system and flow configuration of four-sided lid-driven sinusoidally heated porous cavity

residual method to determine the mixed convection with hydro-magnetic effect in a wavy bottom lid-driven cavity. Wahba [14] has found that there is a critical value of Reynolds number $(R e=129)$ for the four-sided lid-driven problem. The symmetric flow field after this critical value of Reynolds number loses its symmetry to attain an asymmetric flow field. It has also been noted that behind the critical point three possible solutions exist, two of them are stable and asymmetric, while the third solution is unstable and symmetric. Wahba [15] exhibited the time-dependent numerical simulations of 2D unsteady incompressible fluid flow in a four-sided lid-driven cavity using a second-order Crank-Nicolson scheme for a time-dependent term and a compact fourth-order accurate central difference for spatial terms. Fourier power spectrum plots, time history plots for vorticity and stream function, and phase-space trajectories are used to analyze the flow bifurcations inside the cavity.

Literature survey exhibit that the problem of mixed convection in a four-sided lid-driven sinusoidal heated porous cavity has not been investigated numerically. The numerical simulation of the considered problem has been carried out using the stream function-vorticity formulation and Alternating-Direction-Implicit (ADI) finite difference method. Our main goal of the work is to numerically study the mixed convection in a four-sided lid-driven sinusoidal heated porous square cavity. The governing equations are discretized by a finite difference method namely, Alternating-Direction-Implicit (ADI) method to calculate the numerical solutions of the flow variables $\psi, \xi, x$ and $y$ component of velocity, $T$ as well as average Nusselt numbers for different non-dimensional parameters like Grashof number $\left(10^{3} \leq \mathrm{Gr} \leq 10^{5}\right)$, Darcy number $\left(10^{-1} \leq D a \leq 10^{-5}\right)$ and Reynolds number $(10 \leq R e \leq 1000)$ and Prandtl number $(\operatorname{Pr}=0.7)$. The stability and convergence of the numerical scheme have also been obtained by matrix method.

The mathematical formulation of the problem has been discussed in detail in Sect. 2. Section 3 of the article illustrates the numerical discretization of the problem while the stability and the convergence of the numerical scheme used in the paper are presented in Sect. 4. Section 5 and Sect. 6 discuss the code validation and the numerical results of the considered problem.

\section{Mathematical formulation}

\subsection{Physical description}

The physical domain under investigation is a four-sided lid-driven square cavity, with two different cases depending on the direction of the moving walls, depicted in Fig. 1. The top and the bottom walls of the cavity are held at the adiabatic conditions, while the left and right walls of the cavity are held at different temperature conditions. The right wall of the cavity is sinusoidally heated $(T=\operatorname{Sin}(\pi y))$, while the left wall is maintained at a cold temperature $(T=0)$.

Case 1 The top and bottom walls of the cavity are kept in motion from right to left and left to right respectively. 
The left and right walls of the cavity are moving from bottom to top and top to bottom respectively (see Fig. 1a).

Case 2 The top and bottom walls of the cavity are kept in motion from left to right and right to left respectively. The left and right walls of the cavity are moving from top to bottom and bottom to top respectively (see Fig. 1b).

\subsection{Governing equations}

Using the Boussinesq approximations, the governing equations of mixed convection in a sinusoidally heated liddriven porous cavity in non-dimensional form with stream function $(\psi)$ and vorticity $(\xi)[6]$ can be expressed as

$\frac{\partial^{2} \psi}{\partial x^{2}}+\frac{\partial^{2} \psi}{\partial y^{2}}=-\xi$

$$
\begin{gathered}
\frac{\partial \xi}{\partial t}+u \frac{\partial \xi}{\partial x}+v \frac{\partial \xi}{\partial y}-\frac{1}{\operatorname{Re}}\left(\frac{\partial^{2} \xi}{\partial x^{2}}+\frac{\partial^{2} \xi}{\partial y^{2}}\right) \\
+\frac{1}{\operatorname{Re} \mathrm{Da}} \xi-\frac{\mathrm{Gr}}{\operatorname{Re}^{2}} \frac{\partial T}{\partial x}=0
\end{gathered}
$$$$
\frac{\partial T}{\partial t}+u \frac{\partial T}{\partial x}+v \frac{\partial T}{\partial y}-\frac{1}{\operatorname{Re} \operatorname{Pr}}\left(\frac{\partial^{2} T}{\partial x^{2}}+\frac{\partial^{2} T}{\partial y^{2}}\right)=0,
$$$$
u=\frac{\partial \psi}{\partial y}, v=-\frac{\partial \psi}{\partial x}
$$

where

$$
\begin{aligned}
& \xi=\frac{\partial v}{\partial x}-\frac{\partial u}{\partial y}, x=\frac{x^{\prime}}{L}, y=\frac{y^{\prime}}{L}, u=\frac{u^{\prime}}{V_{o}}, v=\frac{v^{\prime}}{V_{o}}, \\
& t=\frac{t^{\prime} V_{o}}{L}, p=\frac{p^{\prime}}{\rho V_{o}^{2}}, T=\frac{T^{\prime}-T_{c}}{\Delta T} .
\end{aligned}
$$

The non-dimensional parameters are

$\operatorname{Pr}=\frac{v}{\alpha}, \quad \mathrm{Da}=\frac{K}{L^{2}}, \quad \operatorname{Re}=\frac{V_{o} L}{v}, \quad \mathrm{Gr}=\frac{g \beta L^{3} \Delta T}{v^{2}}$.

The initial and the boundary conditions associated with the system 1 to 4 for two different cases are described in Tables 1 and 2 respectively.

The heat transfer characteristics across the cavity is generally described by Nusselt number. The local and average Nusselt numbers on the left wall are calculated by

$\mathrm{Nu}_{l}=\left.\frac{\partial T}{\partial x}\right|_{0, j}, \quad \overline{\mathrm{Nu}}{ }_{l}=\int_{0}^{1} \mathrm{Nu}_{l} d y$
Table 1 The initial and the boundary conditions for Case 1

$$
\begin{array}{rrr}
\hline t=0: & u=v=T=\psi=\xi=0 & 0 \leq x, y \leq 1 \\
\mathrm{x}=0: T=0, \psi=0, \frac{\partial \psi}{\partial x}=-0.5 & \xi_{0, j}=\frac{2\left(\psi_{0, j}-\psi_{1, j}+\frac{\partial \psi}{\partial x} I_{0, j} \Delta x\right)}{\Delta x^{2}} \\
t>0: \mathrm{x}=1: & T=\operatorname{Sin}(\pi y), \psi=0, \frac{\partial \psi}{\partial x}=0.5 & \xi_{n, j}=\frac{2\left(\psi_{n, j}-\psi_{n-1, j}-\left.\frac{\partial \psi}{\partial x}\right|_{n, j} \Delta x\right)}{\Delta x^{2}} \\
\mathrm{y}=0: \frac{\partial T}{\partial y}=0, \psi=0, \frac{\partial \psi}{\partial y}=0.5 & \xi_{i, 0}=\frac{2\left(\psi_{i, 0}-\psi_{i, 1}+\frac{\partial \psi}{\partial y} l_{i, 0} \Delta y\right)}{\Delta y^{2}} \\
\mathrm{y}=1: \frac{\partial T}{\partial y}=0, \psi=0, \frac{\partial \psi}{\partial y}=-0.5 & \xi_{i, n}=\frac{2\left(\psi_{i, n}-\psi_{i, n-1}-\left.\frac{\partial \psi}{\partial y}\right|_{i, n} \Delta y\right)}{\Delta y^{2}}
\end{array}
$$

Table 2 The initial and the boundary conditions for Case 2

$$
\begin{aligned}
& t=0: \quad u=v=T=\psi=\xi=0 \quad 0 \leq x, y \leq 1 \\
& \mathrm{x}=0: T=0, \psi=0, \frac{\partial \psi}{\partial x}=0.5 \quad \xi_{0, j}=\frac{2\left(\psi_{0, j}-\psi_{1, j}+\left.\frac{\partial \psi}{\partial x}\right|_{0, j} \Delta x\right)}{\Delta x^{2}} \\
& \mathrm{x}=1: T=\operatorname{Sin}(\pi y), \psi=0, \frac{\partial \psi}{\partial x}=-0.5 \quad \xi_{n, j}=\frac{2\left(\psi_{n, j}-\psi_{n-1, j}-\frac{\partial \psi}{\partial x} I_{n, j} \Delta x\right)}{\Delta x^{2}} \\
& t>0: \mathrm{y}=0: \quad \frac{\partial T}{\partial y}=0, \psi=0, \frac{\partial \psi}{\partial y}=-0.5 \xi_{i, 0}=\frac{2\left(\psi_{i, 0}-\psi_{i, 1}+\frac{\partial \psi}{\partial y} \mathrm{l}_{i, 0} \Delta y\right)}{\Delta y^{2}} \\
& y=1: \frac{\partial T}{\partial y}=0, \psi=0, \frac{\partial \psi}{\partial y}=0.5 \quad \xi_{i, n}=\frac{2\left(\psi_{i, n}-\psi_{i, n-1}-\frac{\partial \psi}{\partial y} l_{i, n} \Delta y\right)}{\Delta y^{2}}
\end{aligned}
$$

\section{Numerical discretization}

In this section, the finite difference method namely, Alternating-Direction-Implicit (ADI) method is used to solve the transient non-dimensional governing equations (1) to (3) subject to the initial and boundary conditions as shown in Tables 1 and 2. The elliptical Poisson equation is discretized by the second-order, central finite difference quotient while the vorticity transport equation and energy equation are solved at each interior nodes using the ADI method in two sweeps. The detailed discretization process of the method is presented in $[1,5]$.

ADI method improves the efficiency of the solution as it solves the parabolic equation in a one-dimensional sense, by keeping the known values for variables or its derivatives in one direction and for other direction we have to calculate the solution of the unknown variables which gives a tri-diagonal system of equations. The LU decomposition method is used to solve the obtained tri-diagonal system of equations. The discretized finite-difference formulation of the vorticity transport equation (2) in both $y$ and $x$ directions respectively, are given by equations (5) and (6).

$$
\begin{aligned}
& A_{i, j}^{t} \xi_{i, j-1}^{t+\frac{1}{2}}+B_{i, j}^{t} \xi_{i, j}^{t+\frac{1}{2}}+C_{i, j}^{t} \xi_{i, j+1}^{t+\frac{1}{2}} \\
& \quad=D_{i, j}^{t} \xi_{i-1, j}^{t}+E_{i, j}^{t} \xi_{i, j}^{t}+F_{i, j}^{t} \xi_{i+1, j}^{t}+G_{i, j}^{t}
\end{aligned}
$$

where the coefficients are defined as follows 


$$
\begin{aligned}
A_{i, j}^{t} & =\left[\frac{\Delta t}{8 \Delta x \Delta y}\left(\psi_{i+1, j}^{t}-\psi_{i-1, j}^{t}\right)-\frac{\Delta t}{2 \operatorname{Re} \Delta y^{2}}\right], \\
B_{i, j}^{t} & =\left[1+\frac{\Delta t}{\operatorname{Re} \Delta y^{2}}\right], \\
C_{i, j}^{t} & =\left[-\frac{\Delta t}{8 \Delta x \Delta y}\left(\psi_{i+1, j}^{t}-\psi_{i-1, j}^{t}\right)-\frac{\Delta t}{2 \operatorname{Re} \Delta y^{2}}\right], \\
D_{i, j}^{t} & =\left[\frac{\Delta t}{8 \Delta x \Delta y}\left(\psi_{i, j+1}^{t}-\psi_{i, j-1}^{t}\right)+\frac{\Delta t}{2 \operatorname{Re} \Delta x^{2}}\right] \\
E_{i, j}^{t} & =\left[1-\frac{\Delta t}{\operatorname{Re} \Delta x^{2}}-\frac{\Delta t}{2 \operatorname{Re} \mathrm{Da}}\right], \\
F_{i, j} & =\left[-\frac{\Delta t}{8 \Delta x \Delta y}\left(\psi_{i, j+1}^{t}-\psi_{i, j-1}^{t}\right)+\frac{\Delta t}{2 \operatorname{Re} \Delta x^{2}}\right] \\
G_{i, j}^{t} & =\frac{G \operatorname{Gr} \Delta t}{4 \operatorname{Re}^{2} \Delta x}\left[T_{i+1, j}^{t}-T_{i-1, j}^{t}\right] \\
H_{i, j}^{t} & \xi_{i-1, j}^{t+1}+I_{i, j}^{t} \xi_{i, j}^{t+1}+J_{i, j}^{t} \xi_{i+1, j}^{t+1}=P_{i, j}^{t} \xi_{i, j-1}^{t+\frac{1}{2}} \\
& +Q_{i, j}^{t} \xi_{i, j}^{t+\frac{1}{2}}+R_{i, j}^{t} \xi_{i, j+1}^{t+\frac{1}{2}}+S_{i, j}^{t}
\end{aligned}
$$

where the coefficients are defined as follows

$$
\begin{aligned}
H_{i, j}^{t} & =\left[-\frac{\Delta t}{8 \Delta x \Delta y}\left(\psi_{i, j+1}^{t}-\psi_{i, j-1}^{t}\right)-\frac{\Delta t}{2 \operatorname{Re} \Delta x^{2}}\right], \\
I_{i, j}^{t} & =\left[1+\frac{\Delta t}{\operatorname{Re} \Delta x^{2}}\right], \\
J_{i, j}^{t} & =\left[\frac{\Delta t}{8 \Delta x \Delta y}\left(\psi_{i, j+1}^{t}-\psi_{i, j-1}^{t}\right)-\frac{\Delta t}{2 \operatorname{Re} \Delta x^{2}}\right], \\
P_{i, j}^{t} & =\left[-\frac{\Delta t}{8 \Delta x \Delta y}\left(\psi_{i+1, j}^{t}-\psi_{i-1, j}^{t}\right)+\frac{\Delta t}{2 \operatorname{Re} \Delta y^{2}}\right] \\
Q_{i, j}^{t} & =\left[1-\frac{\Delta t}{\operatorname{Re} \Delta y^{2}}-\frac{\Delta t}{2 \operatorname{Re} \mathrm{Da}}\right], \\
R_{i, j}^{t} & =\left[\frac{\Delta t}{8 \Delta x \Delta y}\left(\psi_{i+1, j}^{t}-\psi_{i-1, j}^{t}\right)+\frac{\Delta t}{2 \operatorname{Re} \Delta y^{2}}\right] \\
S_{i, j}^{t} & =\frac{\mathrm{Gr} \Delta t}{4 \operatorname{Re}^{2} \Delta x}\left[T_{i+1, j}^{t}-T_{i-1, j}^{t}\right]
\end{aligned}
$$

The finite-difference discretized equations of the energy equation (3) in both the directions can be obtained in a similar fashion. The stream function-vorticity formulation (as described in [1, pp. 467]) is used to solve the discretized equations for obtaining the numerical solutions for the considered problem.

\section{Stability and convergence of the numerical scheme}

This section covers the stability and convergence of the numerical scheme applied to the problem of mixed convection inside a four-sided lid-driven cavity. The following finite-difference approximation formulae for the first and second-order partial derivatives are used to discretize the governing equations.

$D_{+, x}\left(h_{x}\right) \xi_{i, j}=\frac{\xi_{i+1, j}-\xi_{i, j}}{h_{x}}+O\left(h_{x}\right)$

$D_{-, x}\left(h_{x}\right) \xi_{i, j}=\frac{\xi_{i, j}-\xi_{i-1, j}}{h_{x}}+O\left(h_{x}\right)$

$D_{0, x}\left(h_{x}\right) \xi_{i, j}=\frac{\xi_{i+1, j}-\xi_{i-1, j}}{2 h_{x}}+O\left(h_{x}^{2}\right)$,

$D_{0, x}^{2}\left(h_{x}\right) \xi_{i, j}=\frac{\xi_{i+1, j}-2 \xi_{i, j}+\xi_{i-1, j}}{h_{x}^{2}}+O\left(h_{x}^{2}\right)$

$D_{0, y}^{2}\left(h_{y}\right) \xi_{i, j}=\frac{\xi_{i, j+1}-2 \xi_{i, j}+\xi_{i, j-1}}{h_{y}^{2}}+O\left(h_{y}^{2}\right)$

where $h_{x}$ and $h_{y}$ are the grid spacing along $x$ - and $y$-direction respectively. $\Delta t$ is grid spacing along time $(t)$-direction. For simplification of discretization we further assume $A^{*}=D_{0, x^{\prime}}^{2} B^{*}=D_{0, y^{\prime}}^{2} C^{*}=D_{0, x}$ and $D^{*}=D_{0, y}$. Discretizing the vorticity transport equation (2) at time step $t+\frac{1}{2}$ and $t+1$ using the ADI method, we obtain the following system ${ }^{1}$.

$$
\begin{aligned}
\xi_{i, j}^{t+\frac{1}{2}} & =E_{1} \xi_{i, j}^{t}+F_{1} T_{i, j}^{t} \\
E_{1}= & {\left[I+\frac{\Delta t}{2} v_{i, j} D^{*}-\frac{\Delta t}{2 \operatorname{Re}} B^{*}\right]^{-1} } \\
\times & {\left[I-\frac{\Delta t}{2} u_{i, j} C^{*}+\frac{\Delta t}{2 \operatorname{Re}} A^{*}-\frac{\Delta t}{2 \operatorname{Re} \mathrm{Da}}\right] } \\
F_{1}= & {\left[I+\frac{\Delta t}{2} v_{i, j} D^{*}-\frac{\Delta t}{2 \operatorname{Re}} B^{*}\right]^{-1} } \\
\times & {\left[\frac{\Delta t}{2} \frac{G r}{\operatorname{Re}^{2}} C^{*}\right] } \\
\xi_{i, j}^{t+1} & =G_{1} \xi_{i, j}^{t+\frac{1}{2}}+H_{1} T_{i, j}^{t} \\
G_{1} & =\left[I+\frac{\Delta t}{2} u_{i, j} C^{*}-\frac{\Delta t}{2 \operatorname{Re}} A^{*}\right]^{-1} \\
\times & {\left[I-\frac{\Delta t}{2} v_{i, j} D^{*}+\frac{\Delta t}{2 \operatorname{Re}} B^{*}-\frac{\Delta t}{2 \operatorname{Re~Da}}\right] } \\
H_{1} & =\left[I+\frac{\Delta t}{2} u_{i, j} C^{*}-\frac{\Delta t}{2 \operatorname{Re}} A^{*}\right]^{-1} \\
& \times\left[\frac{\Delta t}{2} \frac{G r}{\operatorname{Re}} C^{*}\right]
\end{aligned}
$$

Coupling Eqs. (7) and (8) we get

$$
\begin{aligned}
\xi_{i, j}^{t+1} & =G_{1}\left(E_{1} \xi_{i, j}^{t}+F_{1} T_{i, j}^{t}\right)+H_{1} T_{i, j}^{t} \\
& =G_{1} E_{1} \xi_{i, j}^{t}+\left(G_{1} F_{1}+H_{1}\right) T_{i, j}^{t} .
\end{aligned}
$$

\footnotetext{
${ }_{1}$ For complete discretization process refer [1].
} 
Similarly, on discretizing the energy equation (3) at time step $t+\frac{1}{2}$ and $t+1$, we obtain the following system

$$
\begin{aligned}
& T_{i, j}^{t+\frac{1}{2}}=E_{2} T_{i, j}^{t} \\
& T_{i, j}^{t+1}=G_{2} T_{i, j}^{t+\frac{1}{2}} \\
& T_{i, j}^{t+1}=G_{2} E_{2} T_{i, j}^{t} \\
& E_{2}=\left[I+\frac{\Delta t}{2} v_{i, j} D^{*}-\frac{\Delta t}{2 \operatorname{Re} \operatorname{Pr}} B^{*}\right]^{-1} \\
& \times\left[I-\frac{\Delta t}{2} u_{i, j} C^{*}+\frac{\Delta t}{2 \operatorname{Re} \operatorname{Pr}} A^{*}\right], \\
& G_{2}=\left[I+\frac{\Delta t}{2} u_{i, j} C^{*}-\frac{\Delta t}{2 \operatorname{RePr}} A^{*}\right]^{-1} \\
& \times\left[I-\frac{\Delta t}{2} v_{i, j} D^{*}+\frac{\Delta t}{2 \operatorname{Re} \operatorname{Pr}} B^{*}\right] .
\end{aligned}
$$

Rewriting the system of Eqs. (9) and (12) in matrix form

$$
\left[\begin{array}{c}
\xi_{i, j}^{t+1} \\
T_{i, j}^{t+1}
\end{array}\right]=\left[\begin{array}{cc}
G_{1} E_{1} & G_{1} F_{1}+H_{1} \\
0 & G_{2} E_{2}
\end{array}\right]\left[\begin{array}{c}
\xi_{i, j}^{t} \\
T_{i, j}^{t}
\end{array}\right]
$$

Matrix method requires the norm of the above matrix to be less than or equal to 1 to prove the stability of the considered scheme, i.e.

$\left\|\left[\begin{array}{cc}G_{1} E_{1} & G_{1} F_{1}+H_{1} \\ 0 & G_{2} E_{2}\end{array}\right]\right\| \leq 1$

which require $\left\|G_{1} E_{1}\right\| \leq 1$ and $\left\|G_{2} E_{2}\right\| \leq 1$. To prove $\left\|G_{i} E_{i}\right\| \leq 1$, it is sufficient to show $\rho\left(G_{i}\right) \leq 1$ and $\rho\left(E_{i}\right) \leq 1$ for $i=1$ and 2 . The unconditional stability of the numerical scheme for the considered problem can be obtained by proceeding in the same way as explained by Vusala and Kumar [1].

$\begin{array}{lll}\left|u_{i, j} \operatorname{Re} \Delta x\right| \leq 2, & \left|v_{i, j} \operatorname{Re} \Delta y\right| \leq 2, \quad \Delta t<\operatorname{Re} \Delta x^{2}, \\ \left|u_{i, j} \operatorname{Re} \operatorname{Pr} \Delta x\right| \leq 2, & \left|v_{i, j} \operatorname{Re} \operatorname{Pr} \Delta y\right| \leq 2, \quad \Delta t<\operatorname{Re} \operatorname{Pr} \Delta x^{2} .\end{array}$

The considered scheme is also consistent as local truncation error tends to zero as the step lengths in all direction tends to zeros. Therefore, the considered scheme is convergent by Lax's equivalence theorem [12, pp.72].

\section{Code validation}

\begin{tabular}{|c|c|c|c|c|c|}
\hline Cases & & Da & $\psi$ & $\xi$ & $T$ \\
\hline \multirow[t]{4}{*}{ I } & $\begin{array}{l}\text { Chattopadhyay } \\
\text { et al. [6] }\end{array}$ & 0.001 & 0.03144 & 0.74462 & 0.33653 \\
\hline & Present & 0.001 & 0.03146 & 0.74465 & 0.33655 \\
\hline & $\begin{array}{l}\text { Chattopadhyay } \\
\text { et al. [6] }\end{array}$ & 0.01 & 0.22881 & 5.40223 & 0.34412 \\
\hline & Present & 0.01 & 0.22887 & 5.40229 & 0.34414 \\
\hline \multirow[t]{4}{*}{ II } & $\begin{array}{l}\text { Chattopadhyay } \\
\text { et al. [6] }\end{array}$ & 0.001 & 0.06290 & 0.74297 & 0.33696 \\
\hline & Present & 0.001 & 0.06288 & 0.74294 & 0.33692 \\
\hline & $\begin{array}{l}\text { Chattopadhyay } \\
\text { et al. [6] }\end{array}$ & 0.01 & 0.30975 & 5.23297 & 0.34678 \\
\hline & Present & 0.01 & 0.30970 & 5.23290 & 0.34678 \\
\hline \multirow[t]{4}{*}{ III } & $\begin{array}{l}\text { Chattopadhyay } \\
\text { et al. [6] }\end{array}$ & 0.001 & 0.04724 & 0.74571 & 0.33852 \\
\hline & Present & 0.001 & 0.04724 & 0.74569 & 0.33855 \\
\hline & $\begin{array}{l}\text { Chattopadhyay } \\
\text { et al. [6] }\end{array}$ & 0.01 & 0.27275 & 5.44283 & 0.36841 \\
\hline & Present & 0.01 & 0.27272 & 5.44288 & 0.36845 \\
\hline
\end{tabular}

We have verified our computer code by comparing the results of problem mixed convection inside a double lid-driven square cavity investigated by Chattopadhyay et al. [6]. They have examined three different cases
Table 3 Comparision of Stream function $(\psi)$, vorticity $(\xi)$ and temperature $(T)$ at midpoint of the cavity for $\phi=0, R i=100$ and different Darcy numbers

depending upon the direction of the moving walls. We have compared the streamline and isotherm contours with the first case studied by Chattopadhyay et al. [6]. In the first case, the left and right vertical wall of the cavity are moving from bottom to top and top to bottom respectively with a uniform speed. We have found that our results are in good agreement with those of [6] (see Figs. 2, 3). Comparison of the numerical values of streamfunction $(\psi)$, vorticity $(\xi)$, and temperature $(T)$ at midpoint of the cavity for all the three different cases discussed by Chattopadhyay et al. [6] for $\phi=0, R i=100$ with different Darcy numbers is given in Table 3 with $81 \times 81$ grid.

\section{Results and discussion}

A numerical study has been performed to investigate the mixed convection inside a four-sided lid-driven porous square cavity, whose top and bottom walls are adiabatic. The left wall of the cavity kept at a cold temperature while the right wall with sinusoidal temperature. The fluid is chosen with Prandtl number 0.7 and will be kept fixed throughout the numerical computations. The numerical results are calculated for two different cases according to the direction of moving walls as described in Fig. 1 with different values of non-dimensional parameters such as $\mathrm{Gr}$, $\mathrm{Da}$, and Re. We have analyzed the effect of Grashof number, Darcy number, and Reynolds number on streamline contours, isotherm contours, horizontal and vertical velocities, temperature along the vertical and horizontal line through 


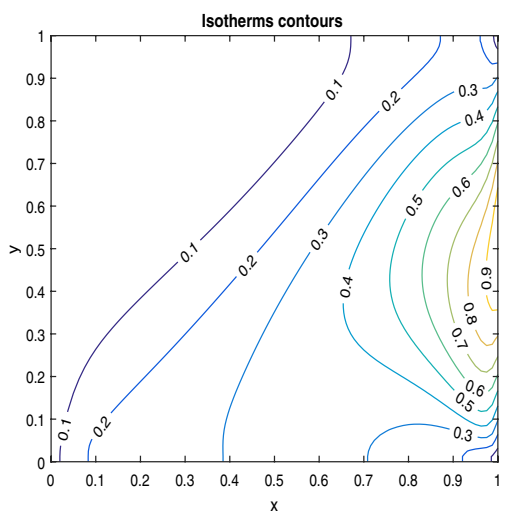

$\mathrm{Da}=0.001$

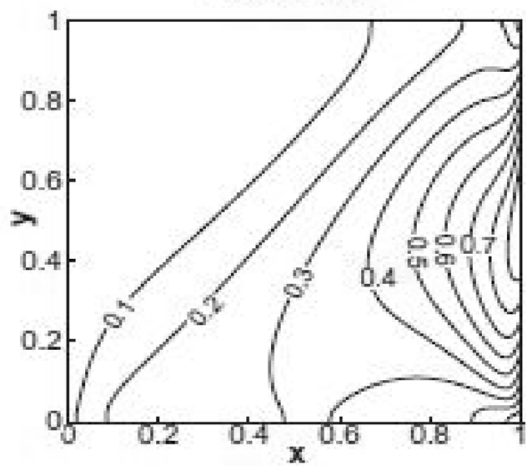

(a) $R i=0.1$

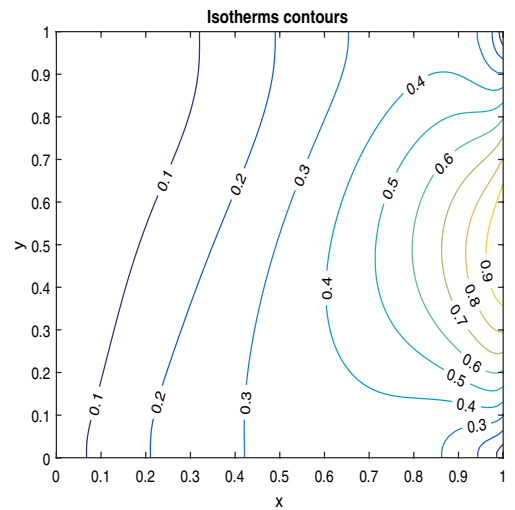

$\mathrm{Da}=0.001$

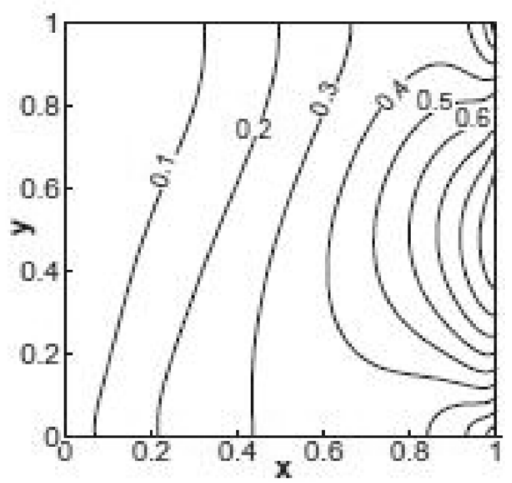

(b) $R i=1$

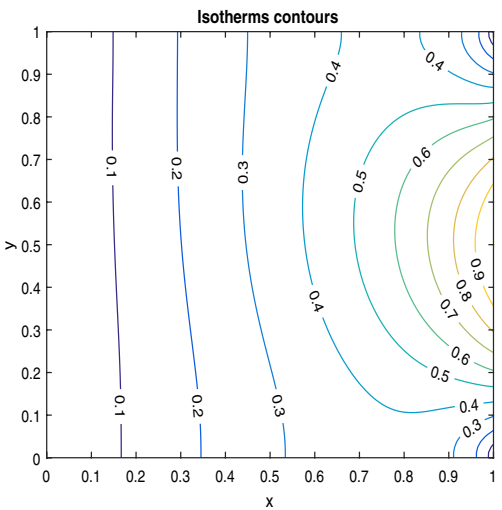

$\mathrm{Da}=0.001$

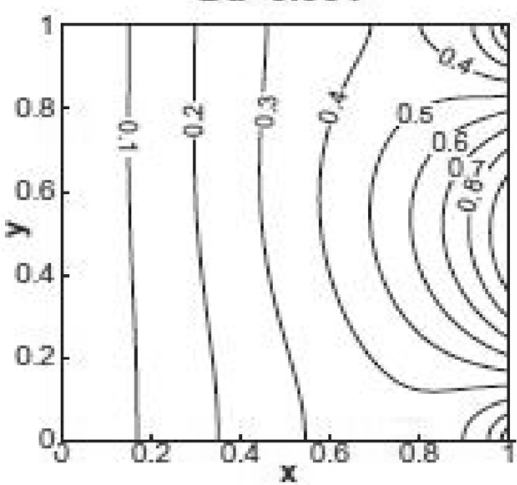

(c) $R i=100$

Fig. 2 Comparison of isotherm contours in top (present) and bottom (Chattopadhyay et al. [6]) with $\operatorname{Pr}=0.7, \mathrm{Da}=0.001$ and $\mathrm{Gr}=10^{4}$, for different Richardson numbers $\mathbf{a} R i=0.1, \mathbf{b} R i=1, \mathbf{c} R i=100$

the geometric center of the square cavity respectively, and average Nusselt number along the left wall.

In order to study the effect of Grashof number (Gr), it is varied from $10^{3}$ to $10^{5}$ with $\operatorname{Re}=100$, and $\mathrm{Da}=10^{-3}$. The Darcy number $(\mathrm{Da})$ is ranged from $10^{-1}$ to $10^{5}$ for $\mathrm{Re}=100$ and $\mathrm{Gr}=10^{3}$ to investigate its effect. The Reynolds number (Re) varies from 10 to 1000 with $\mathrm{Da}=10^{-3}$ and $\mathrm{Gr}=10^{3} \mathrm{for}$ the effect of Reynolds number.

\subsection{Case 1}

In this case, the left wall of the square cavity is maintained at a cold temperature and is moving upward while the right wall is kept at a hot sinusoidal temperature condition and is moving in a downward direction. The top and bottom walls of the cavity are adiabatic. The top and bottom walls move from right to left and left to right at a uniform speed respectively.

\subsubsection{Effect of Grashof number}

Under this subsection, we have presented the numerical results with the effect of the Grashof number. In Figs. 4 and 5, we have displayed the streamline and isotherm contours for three different values of Grashof number namely $\mathrm{Gr}=10^{3}, 10^{4}$ and $10^{5}$ with $\operatorname{Pr}=0.7, \operatorname{Re}=100$ and $\mathrm{Da}=10^{-3}$. Visual examination of the streamline and isotherm contours exhibits that there is a vital change among the streamline and isotherm contours for fixed $\mathrm{Da}$, Re with different $\mathrm{Gr}$ numbers.

The four streamline contours have developed in the vicinity of the moving walls at $\mathrm{Gr}=10^{3}$ and $10^{4}$ (see Fig. 4 a, b). These contours are almost symmetrically opposite with respect to the vertical line through the geometric center of the square cavity. It signifies that the darcy effect is very less for the dominant regimes of forced convection. A remarkable change occurs in the streamline contours as the Grashof number increases from $10^{4}$ to $10^{5}$. Two weaker clockwise rotating streamline contours are generated in the vicinity of vertical sidewalls in addition to a single anticlockwise flow circulation in the middle of the cavity 

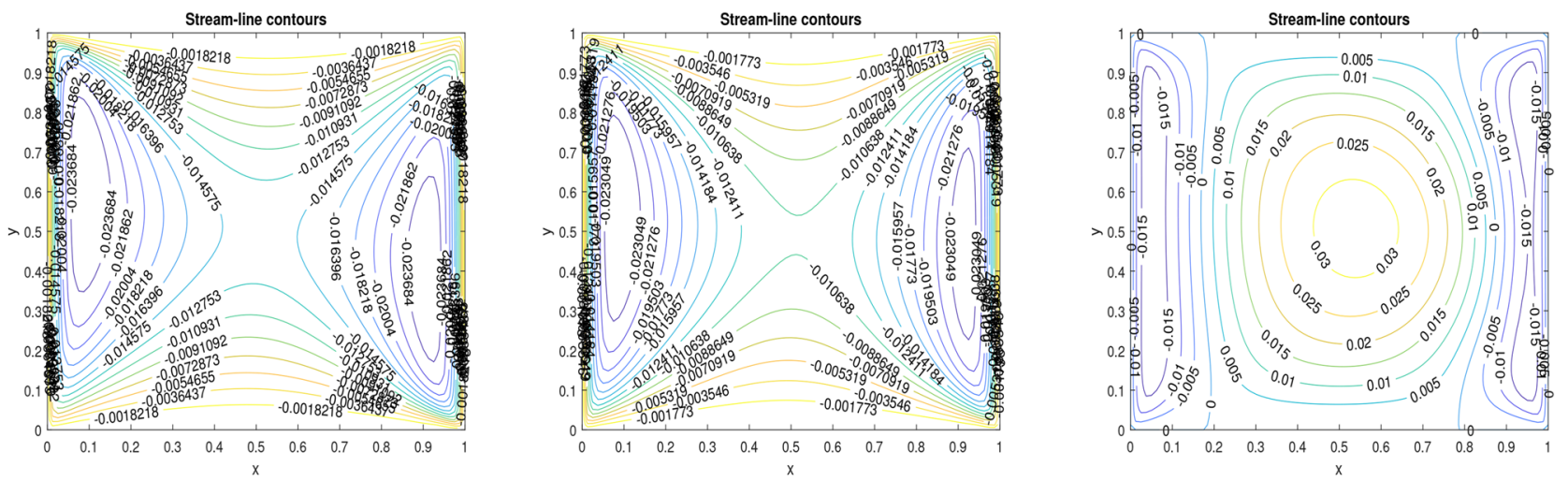

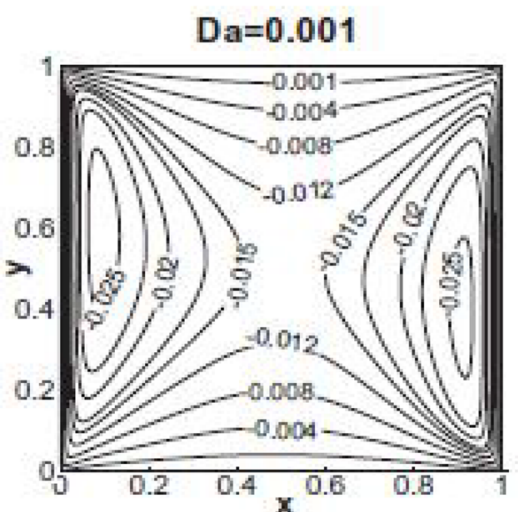

(a) $R i=0.1$

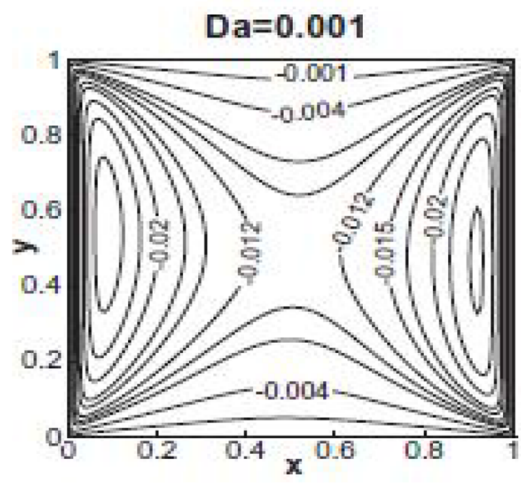

(b) $R i=1$

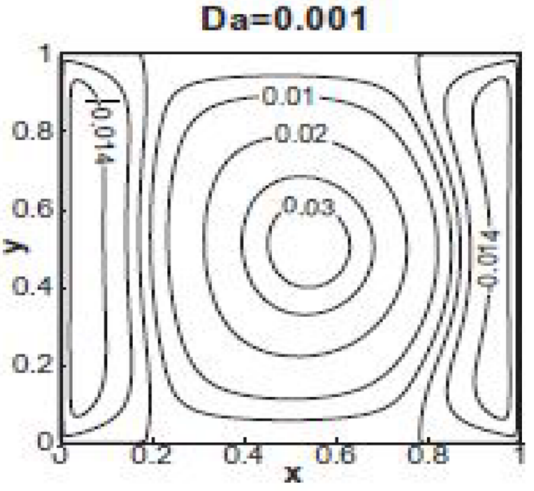

(c) $R i=100$

Fig. 3 Comparison of streamline contours in top (present) and bottom (Chattopadhyay et al. [6]) with $\mathrm{Pr}=0.7, \mathrm{Da}=0.001$ and $\mathrm{Gr}=10^{4}$ for different Richardson numbers. $\mathbf{a} R i=0.1, \mathbf{b} R i=1, \mathbf{c} R i=100$

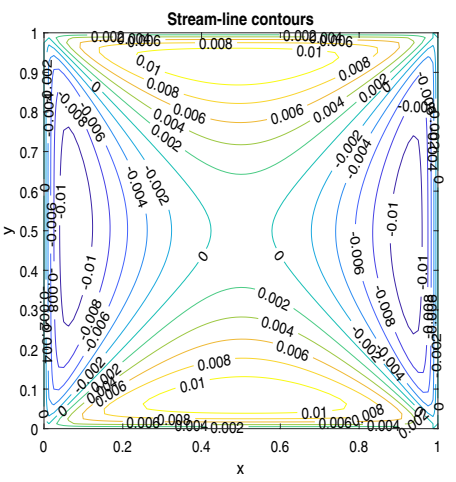

(a) $G r=10^{3}$

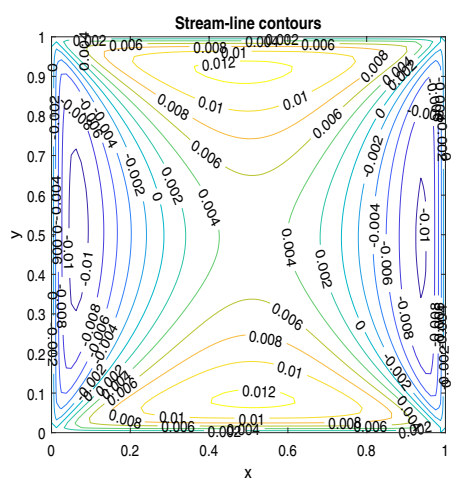

(b) $G r=10^{4}$

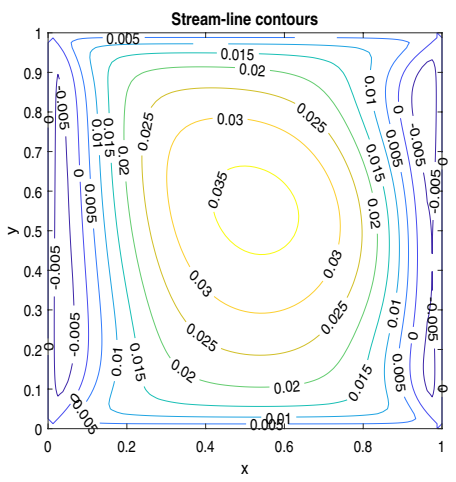

(c) $G r=10^{5}$

Fig. 4 Streamline contours for $\operatorname{Pr}=0.7, \operatorname{Re}=100, \mathrm{Da}=10^{-3}$ at different values of Grashof number. $\mathbf{a} \mathrm{Gr}=10^{3}, \mathbf{b ~ G r}=10^{4}$, c Gr$=10^{5}$, for case 1

at $\mathrm{Gr}=10^{5}$ (see Fig. 4c). Streamline contours in the vicinity of the vertical walls come closer together which forms the regions of the high normal velocity gradient. Due to the motion of the walls, these circular streamline contours are stretched along the primary diagonal of the square cavity. The absolute value of stream function increases with an increase of Grashof number $\mathrm{Gr}=10^{3}$ to $10^{5}$, and 


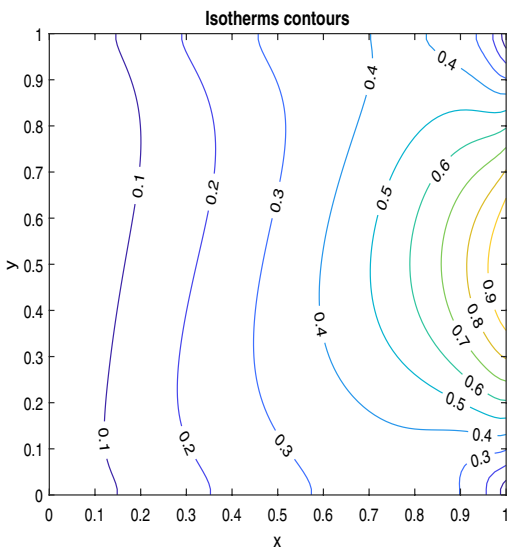

(a) $G r=10^{3}$

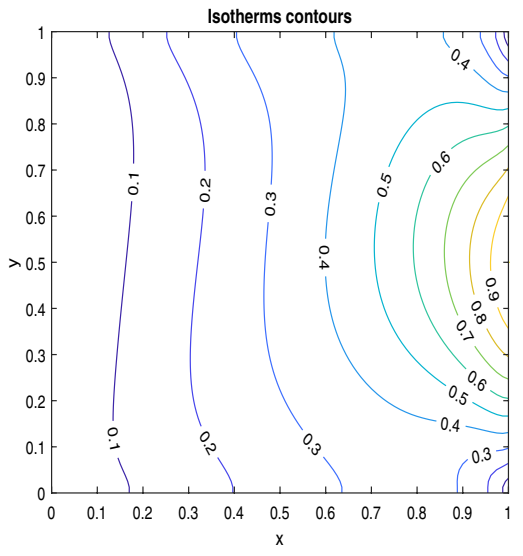

(b) $G r=10^{4}$

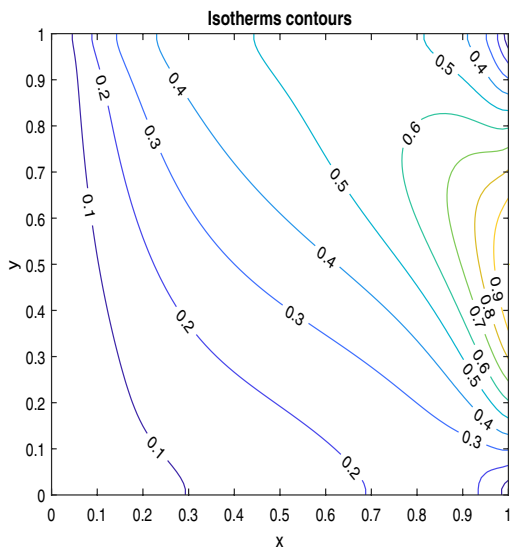

(c) $G r=10^{5}$

Fig. 5 Isotherm contours for $\operatorname{Pr}=0.7, \operatorname{Re}=100, \mathrm{Da}=10^{-3}$ at different values of Grashof number, $\mathbf{a} \mathrm{Gr}=10^{3}, \mathbf{b} \mathrm{Gr}=10^{4}, \mathbf{c} \mathrm{Gr}=10^{5}$, for case

has a maximum around the center of the square cavity at $\mathrm{Gr}=10^{5}$. An increase in the Grashof number produces the flow activity more inward into the cavity, which prompts more energy to be transferred away from the sliding walls, thus causing notable changes in the flow behavior.

Figure $5 a, b$ show that the isotherm contours are symmetric about the horizontal line through the geometric center of the square cavity for Grashof number $\mathrm{Gr}=10^{3}$ and $10^{4}$, while it becomes asymmetric for $\mathrm{Gr}=10^{5}$. The behavior of isotherm contours are almost similar for $\mathrm{Gr}=10^{3}$ and $10^{4}$, but the isotherm pattern changes very rapidly with an increase of $\mathrm{Gr}=10^{4}$ to $10^{5}$. Since the sinusoidal temperature distribution is imposed on the right wall and the left wall is cold, the heat starts to transfer from the right wall to the left wall in an oscillating manner for $\mathrm{Gr}=10^{3}, 10^{4}$ (see Fig. 5a, b), while it starts shifting towards the left-top corner of the cavity with an increase of $\mathrm{Gr}=10^{4}$ to $10^{5}$ (see Fig. $5 \mathrm{c}$ ).

Figure 6 illustrates the effect of Grashof number on $u$-velocity along the vertical center-line, $v$-velocity, and temperature along the horizontal center-line of the cavity, and average Nusselt number along the left vertical wall of the square cavity by ranging $\mathrm{Gr}=10^{3}$ to $10^{5}$. The $u$-velocity and $v$-velocity are symmetric about the horizontal and vertical centerline of the cavity respectively, as described in Fig. $6 a$, b. The absolute value of velocity is maximum near the vicinity of moving walls. The absolute value of velocity increases with an increase of Grashof number $\mathrm{Gr}=10^{3}$ to $10^{5}$. Figure $6 \mathrm{c}$ demonstrates that the temperature profiles increase smoothly from the cold (left) wall to the hot sinusoidal (right) wall along the horizontal center-line of the cavity for $\mathrm{Gr}=10^{3}$ to $10^{5}$. The temperature profiles increase from cold wall to hot wall in an oscillating manner for Grashof number $\mathrm{Gr}=10^{5}$. The average Nusselt number along the left vertical wall of the cavity increases with time and attain its steady-state solution after $t=30$, (see Fig. $6 \mathrm{~d}$ ). Table 4 shows that the average Nusselt number increases with an increase of Grashof number from $\mathrm{Gr}=10^{3}$ to $10^{5}$.

\subsubsection{Effect of Darcy number}

In this subsection, we will examine the effect of Darcy number on the streamline, isotherm, $u$-velocity, $v$-velocity, temperature, and average Nusselt number. The streamline and isotherm contours for different values of Darcy number ranging from $10^{-1}$ to $10^{-5}$ with $\mathrm{Re}=100, \mathrm{Gr}=10^{3}$ are described respectively in Figs. 7 and 8 . Figure $7 a-e$ show that the four streamline contours are generated in the vicinity of the moving walls especially Left Primary Vortex (LPV), Right Primary Vortex (RPV), Top Primary Vortex (TPV), and Bottom Primary Vortex (BPV). These primary vortices display the stream function values along with a negative or positive sign. A positive sign signifies that the vortex is moving in an anti-clockwise direction, while a negative sign indicates a clockwise direction of the vortex. The flow circulation of these primary vortices become negligible in the middle of the square cavity with a decrease in Darcy number from $10^{-1}$ to $10^{-5}$. The stream function values at TPV are $0.030,0.025,0.010,0.003$, and 0.0006 for Darcy number $10^{-1}, 10^{-2}, 10^{-3}, 10^{-4}$, and $10^{-5}$ respectively, which illustrates that the magnitude decreases with a decrease of Darcy number.

The location of the centers of these primary vortices are represented in Table 5 for different Darcy numbers. The center of TPV begins moving to the right and slightly upward which is at $(0.5000,0.9687)$ and the RPV starts shifting upward and slightly to the right at $(0.9687,0.5000)$ as Darcy number decreases from $\mathrm{Da}=10^{-1}$ to $10^{-5}$. The 


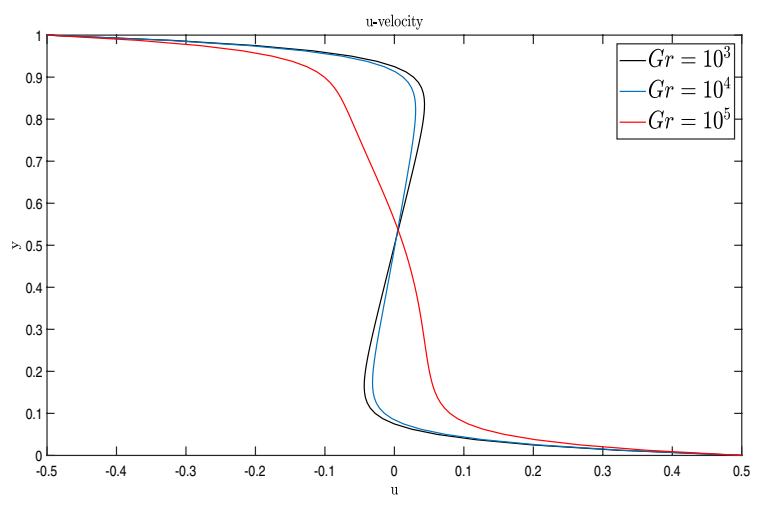

(a) $u$-velocity

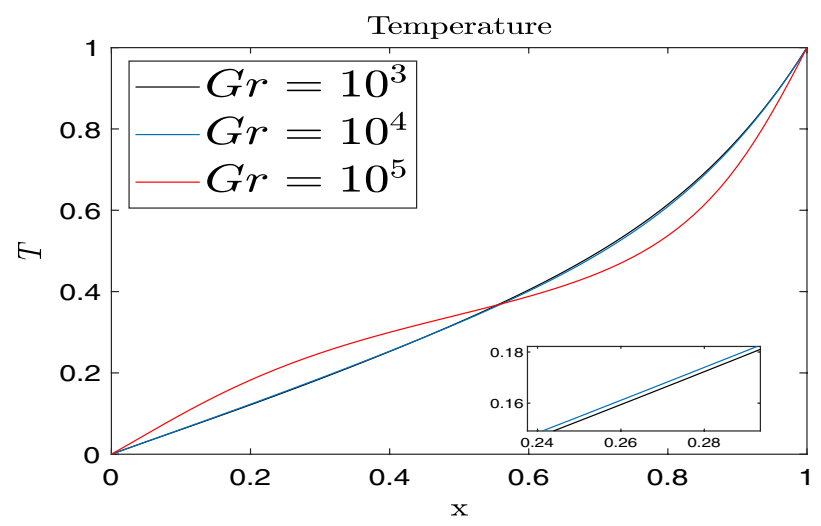

(c) Temperature $(T)$

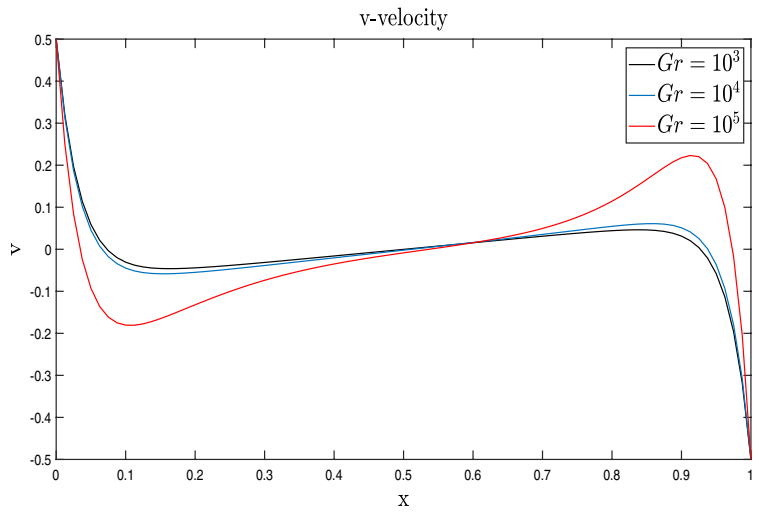

(b) $v$-velocity

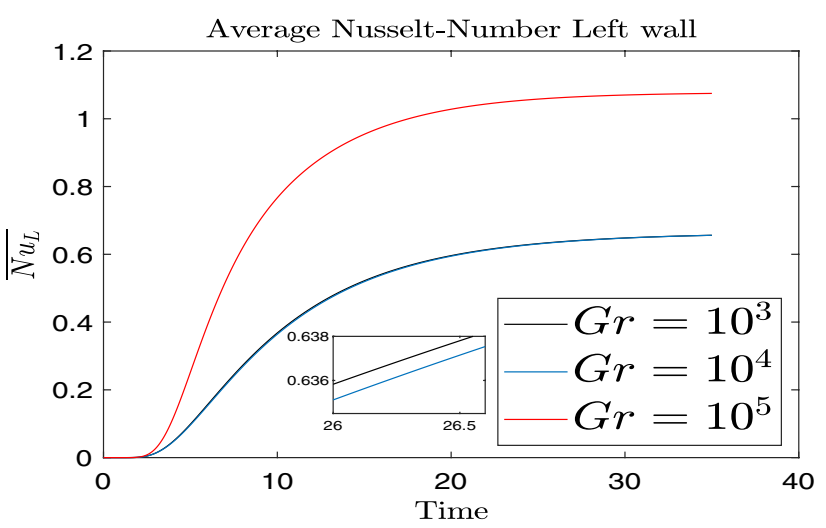

(d) Average Nusselt Number $\left(\overline{N u_{l}}\right)$

Fig. 6 Effect of Grashof numbers on a $u$-velocity, b $v$-velocity, c temperature $(T)$, d average Nusselt number on left wall with $\operatorname{Pr}=0.7, \operatorname{Re}=100, \mathrm{Da}=10^{-3}$, for case 1

Table 4 Average Nusselt number along the left vertical wall of the cavity at different Grashof numbers for Case 1

\begin{tabular}{lll}
$\overline{\overline{\mathrm{Nu}}}$ & & \\
\hline $\mathrm{Gr}=10^{3}$ & $\mathrm{Gr}=10^{4}$ & $\mathrm{Gr}=10^{5}$ \\
\hline 0.6561 & 0.6561 & 1.075 \\
\hline
\end{tabular}

center of BPV begins moving to the left and slightly downward which is at $(0.5000,0.0313)$, and the LPV start moving towards the left and slightly downward at $(0.0313,0.5000)$ as Darcy number decreases from $\mathrm{Da}=10^{-1}$ to $10^{-5}$. It is found that the four streamline contours start shifting toward the vicinity of their concerned walls with a decrease of Darcy number.

The effect of Darcy number on the isotherm contours for the considered problem is examined in Fig. 8a-e by ranging $\mathrm{Da}$ from $10^{-1}$ to $10^{-5}$ with $\mathrm{Re}=100$ and $\mathrm{Gr}=10^{3}$. The isotherm contours are almost the same for different Da numbers except for the amplitude of the oscillation. The isotherm contours look like concentric circles on the right wall of the cavity. In addition to these contours, two small isotherm contours also generated in the vicinity of the top, bottom-right wall. The isotherm contours attain the sinusoidal curve as it moves towards the left wall of the cavity. The oscillation of these sinusoidal curves reduces with a decrease of Darcy number from $\mathrm{Da}=10^{-1}$ to $10^{-5}$.

Figure 9 illustrates the effect of Darcy number on the velocity profiles, temperature, and the average Nusselt number by ranging $\mathrm{Da}=10^{-1}$ to $\mathrm{Da}=10^{-5}$. There is an enormous difference in the velocity profiles in the middle of the square cavity for different Darcy numbers. The direction and magnitude of the velocity changes due to the fluid circulation inside the cavity, and it is maximum the vicinity of the moving walls (see Fig. 9a, b). The absolute value of the fluid velocity along the horizontal and vertical line through the midpoint of the cavity decreases with a decrease of Darcy number from $\mathrm{Da}=10^{-1}$ to $10^{-5}$. The temperature profiles along the horizontal centerline of the cavity increase with a decrease of darcy number $\mathrm{Da}=10^{-1}$ to $\mathrm{Da}=10^{-5}$, as illustrated in Fig. 9c. Figure $9 \mathrm{~d}$ and Table 6 show that the average Nusselt number along the left wall of the cavity decreases with a decrease of Darcy number. 


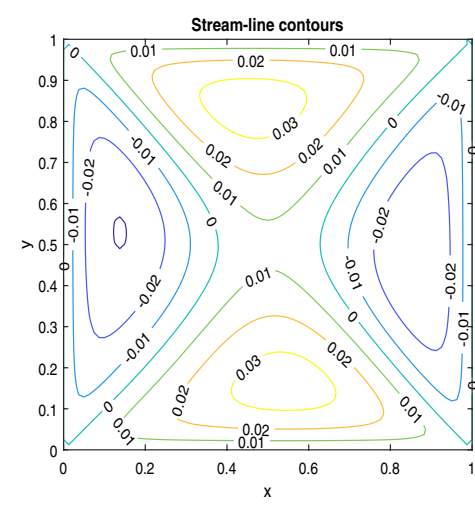

(a) $D a=10^{-1}$

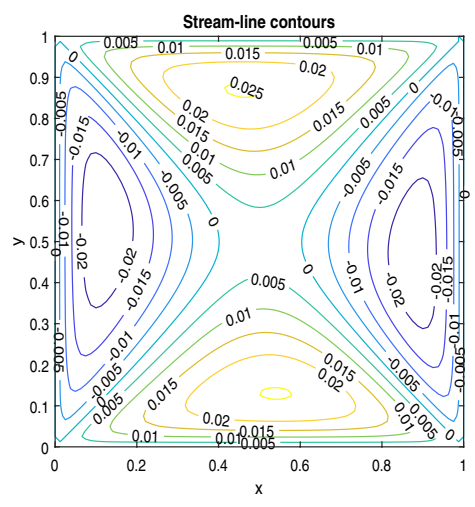

(b) $D a=10^{-2}$

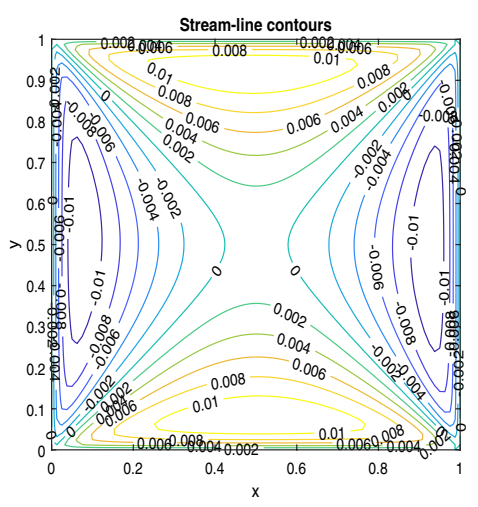

(c) $D a=10^{-3}$

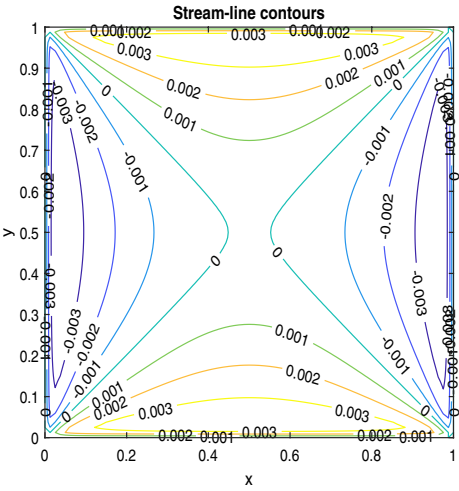

(d) $D a=10^{-4}$

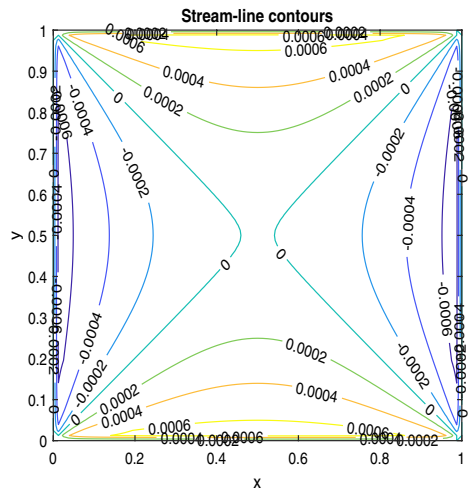

(e) $D a=10^{-5}$

Fig. 7 Streamline contours for $\operatorname{Pr}=0.7, \operatorname{Re}=100, \mathrm{Gr}=10^{3}$ at different values of Darcy number. $\mathbf{a} \mathrm{Da}=10^{-1}, \mathbf{b ~ D a}=10^{-2}, \mathbf{c ~ D a}=10^{-3}, \mathbf{d}$ $\mathrm{Da}=10^{-4}, \mathrm{e} \mathrm{Da}=10^{-5}$, for case 1

Table 5 Locations of the centers of the streamline vortices for Case 1 at different Darcy numbers

\begin{tabular}{lllll}
\hline Darcy number & Top primary vortex (TPV) & $\begin{array}{l}\text { Bottom } \\
\text { primary vortex } \\
\text { (BPV) }\end{array}$ & $\begin{array}{l}\text { Left primary vortex (LPV) } \\
\text { Right primary } \\
\text { vortex (RPV) }\end{array}$ \\
\hline $10^{-1}$ & $0.4625,0.8375$ & $0.5375,0.1625$ & $0.1375,0.5375$ & $0.8375,0.4750$ \\
$10^{-2}$ & $0.4625,0.8625$ & $0.5375,0.1250$ & $0.1250,0.5375$ & $0.8625,0.4750$ \\
$10^{-3}$ & $0.4875,0.9125$ & $0.5125,0.0750$ & $0.0750,0.5125$ & $0.9250,0.4875$ \\
$10^{-4}$ & $0.5000,0.9412$ & $0.5000,0.0600$ & $0.0600,0.5000$ & $0.9412,0.5000$ \\
$10^{-5}$ & $0.5000,0.9687$ & $0.5000,0.0313$ & $0.0313,0.5000$ & $0.9687,0.5000$ \\
\hline
\end{tabular}

\subsubsection{Effect of Reynolds number}

Figure 10 illustrates that four streamline contours are generated in the vicinity of the moving walls, and these contours shift slightly in the direction of moving walls with an increase of Reynolds number $\mathrm{Re}=10-1000$. The streamline contours in the vicinity of vertical walls move in an anticlockwise direction, and then followed by streamline contours moving in a clockwise direction in the middle of the cavity at $\operatorname{Re}=10$. Figure 11 shows that the oscillation of isotherm contours near the left wall of the cavity increases with an increase of Reynolds number $\mathrm{Re}=10$ to 1000 . The heat transfer from the right wall to the left wall of the cavity decreases significantly with a rise of Reynolds number from $R e=10$ to 1000 .

Figure 12 demonstrates the effect of Reynolds number on the velocity, temperature, and the average Nusselt number for different Reynolds $(\operatorname{Re}=10,50,150,400,1000)$ number and $\mathrm{Da}=10^{-3}, \mathrm{Gr}=10^{3}$. The velocity profiles are almost zero except in the vicinity of the moving walls for 


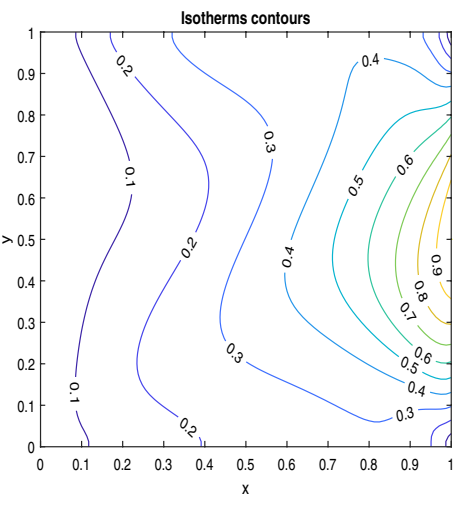

(a) $D a=10^{-1}$

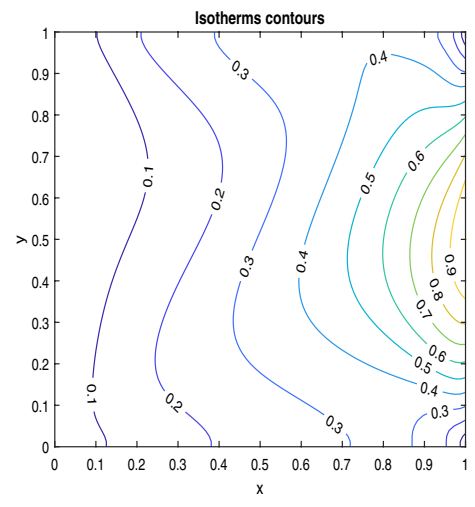

(b) $D a=10^{-2}$

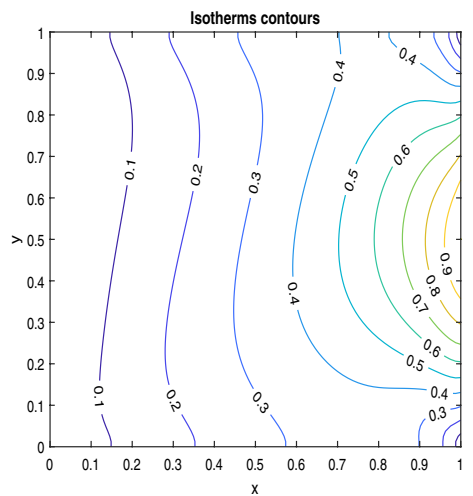

(c) $D a=10^{-3}$

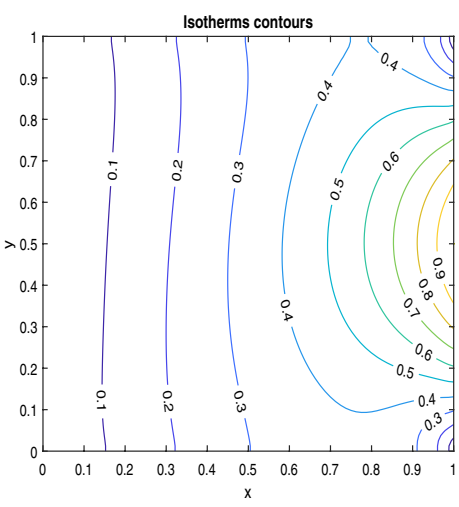

(d) $D a=10^{-4}$

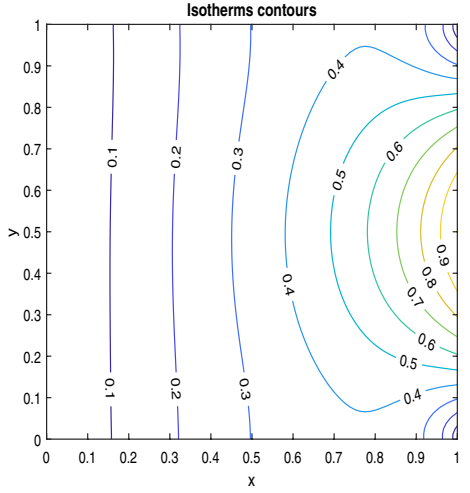

(e) $D a=10^{-5}$

Fig. 8 Isotherm contours for $\operatorname{Pr}=0.7, \operatorname{Re}=100, \mathrm{Gr}=10^{3}$ at different values of Darcy number. $\mathbf{a} \mathrm{Da}=10^{-1}, \mathbf{b} \mathrm{Da}=10^{-2}, \mathbf{c} \mathrm{Da}=10^{-3}, \mathbf{d}$ $\mathrm{Da}=10^{-4}, \mathbf{e D a}=10^{-5}$, for case 1

$\operatorname{Re}=10$ to 1000 . The varations in velocity can be observed as we move from $\operatorname{Re}=10$ to 50 . But as we move from $\operatorname{Re}=50$ to 1000 the varations are insignificant (see Fig. 12a, b).

The temperature profiles along the horizontal centerline of the square cavity decreases with an increase of Reynolds number from $\operatorname{Re}=10$ to 1000 , as depicted in Fig. 12c. Figure $12 \mathrm{~d}$ shows that the average Nusselt number along the left vertical wall of the cavity varies with the Reynolds numbers. The rate of change of the average Nusselt number is greater for smaller value of the Reynolds number before the study state is attained. The average Nusselt number increases with an increase of Reynolds number from $\mathrm{Re}=10$ to 1000 .

\subsection{Case 2}

In this case, the right wall is kept at a hot sinusoidal temperature condition and is moving in an upward direction while the left wall of the square cavity is maintained at a cold temperature and is moving downward. The top and bottom wall of the cavity are adiabatic and move from left to right, and right to left at a uniform speed respectively.

\subsubsection{Effect of Grashof number}

The effect of Grashof number on the streamline and isotherm contours is presented in Figs. 13 and 14 respectively, by ranging $\mathrm{Gr}=10^{3}$ to $10^{5}$ and $\mathrm{Re}=100, \mathrm{Da}=10^{-3}$. Figure $13 a, b$ show that the four streamline contours are generated in the vicinity of the moving walls of the cavity at Grashof number $\mathrm{Gr}=10^{3}$ and $10^{4}$, similar to Case 1 as discussed before. The flow circulation of these four contours is negligible in the middle of the cavity. The two small contours in the vicinity of the top and the bottom wall of the cavity are generated at $\mathrm{Gr}=10^{5}$, as depicted in Fig. 13c. A single elliptic type contour in the middle of the square cavity is also developed together with these contours. Although the flow directions of these contours are the same as in Case 1, the exception is in the positions of these contours. The elliptic streamline contour of value 0.035 is displaced towards the right vertical wall of 


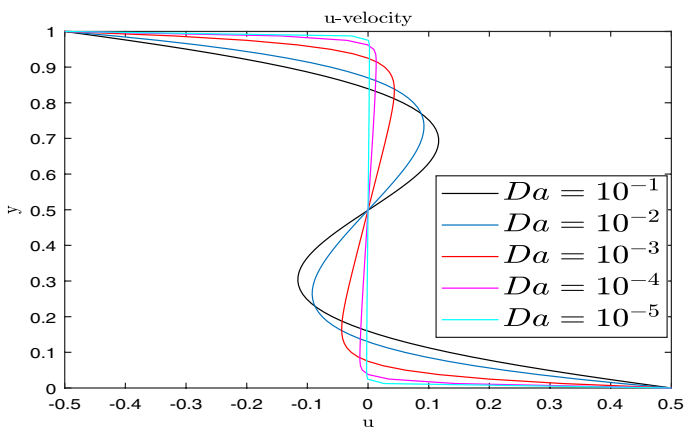

(a) $u$-velocity

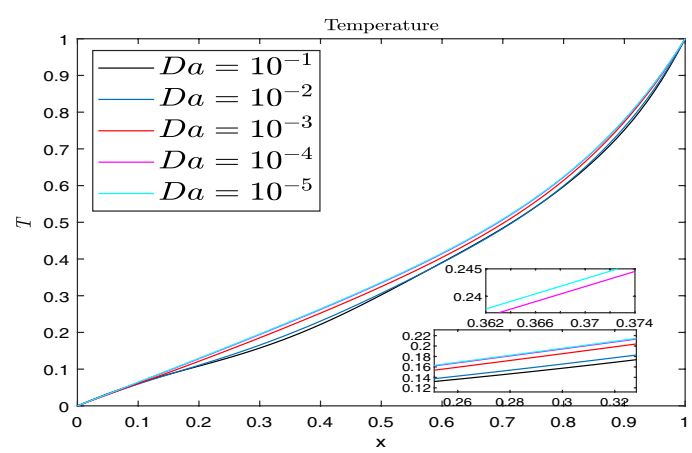

(c) Temperature $(T)$

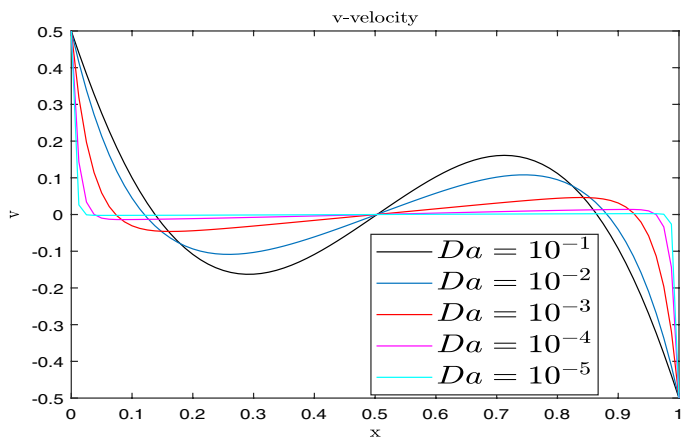

(b) $v$-velocity

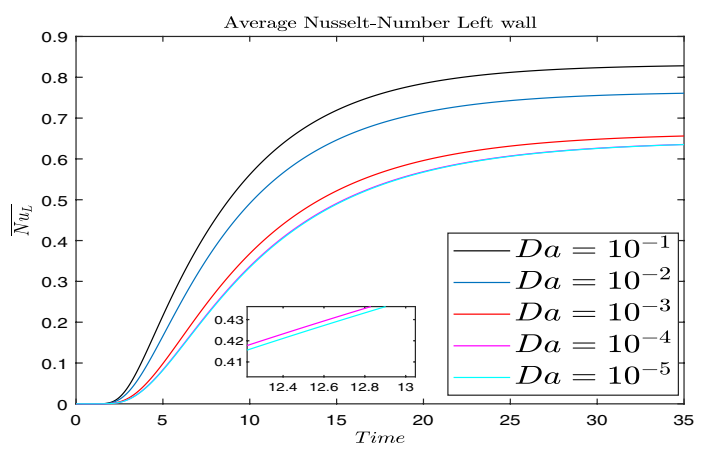

(d) Average Nusselt Number $\left(\overline{N u_{l}}\right)$

Fig. 9 Effect of Darcy numbers on a $u$-velocity, b $v$-velocity, c temperature $(T)$, d average Nusselt Number on left wall with $\operatorname{Pr}=0.7, \operatorname{Re}=100, \mathrm{Gr}=10^{3}$, for case 1

Table 6 Average Nusselt number along the left vertical wall of the cavity at different Darcy numbers for Case 1

\begin{tabular}{lllll}
$\overline{\overline{\mathrm{Nu}}}$ & & & & \\
\hline $\mathrm{Da}=10^{-1}$ & $\mathrm{Da}=10^{-2}$ & $\mathrm{Da}=10^{-3}$ & $\mathrm{Da}=10^{-4}$ & $\mathrm{Da}=10^{-5}$ \\
\hline 0.8281 & 0.7609 & 0.6561 & 0.6354 & 0.6351 \\
\hline
\end{tabular}

the cavity at $\mathrm{Gr}=10^{5}$. The absolute value of stream function increases with an increase of Grashof number ranging from $10^{3}$ to $10^{5}$.

Figure 14 shows that the isotherm profiles for the considered problem are almost similar to Case 1, as discussed before. But due to the opposite motions of the walls as compared to Case 1, the isotherm contours of oscillating sinusoidal curve in the vicinity of the left vertical wall first decrease then increase and again decrease from the top wall to the bottom wall of the cavity at $\mathrm{Gr}=10^{3}$ and $10^{4}$ (see Fig. 14a, b). There is a rapid change in the isotherm profiles at $\mathrm{Gr}=10^{5}$ (see Fig. 14c), in the vicinity of the left wall of the cavity. The amplitude of the isotherm contours shifting from the right wall to the left top wall of the cavity is higher than Case 1.
Figure 15 presents the effect of Grashof number on the velocity profile, temperature along the horizontal centerline of the cavity, and average Nusselt number along the left vertical wall of the cavity by ranging $\mathrm{Gr}$ from $10^{3}$ to $10^{5}$ and fixed $\mathrm{Re}=100, \mathrm{Da}=10^{-3}$. The absolute value of the velocity in the vicinity of the moving walls decreases with an increase of Grashof number (see Fig. 15a, b). The temperature profiles behave alike Case 1, as illustrated in Fig. 15c. Table 7 exhibits that the average Nusselt number rises with an increase of Grashof number from $10^{3}$ to $10^{5}$.

\subsubsection{Effect of Darcy number}

Figures 16 and 17 respectively illustrate the effect of Darcy number for the considered problem on the streamline and isotherm contours by ranging Da from $10^{-1}$ to $10^{-5}$ and for fixed $\operatorname{Re}=100, \mathrm{Gr}=10^{3}$. The four streamline contours which are symmetric about the horizontal and vertical centerline of the cavity are generated for different Darcy numbers. Table 8 shows that the center of TPV starts shifting slightly to the right and in an upward direction which is at $(0.5400,0.9608)$, while the BPV starts moving to the right and in a downward direction at $(0.5000,0.0212)$ with a decrease of Darcy number from $10^{-1}$ to $10^{-5}$. This behavior 


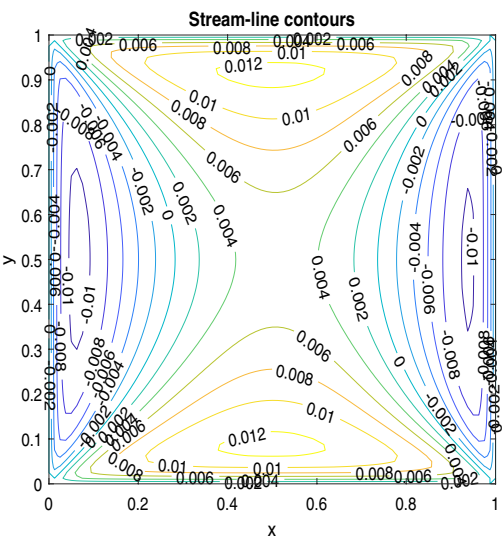

(a) $R e=10$

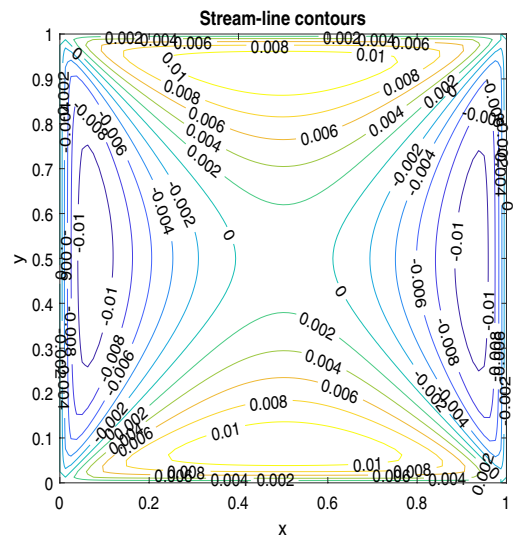

(b) $R e=50$

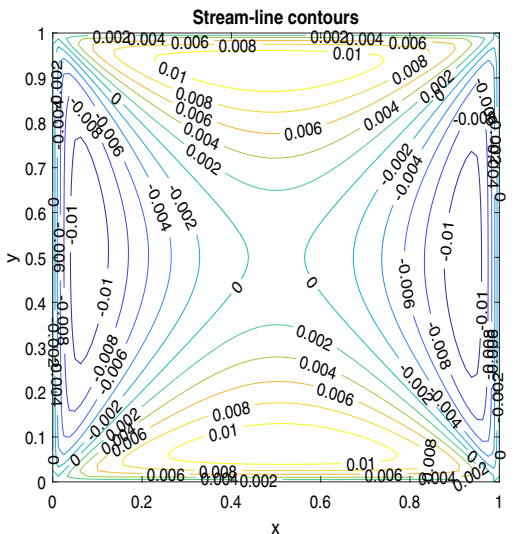

(c) $R e=150$

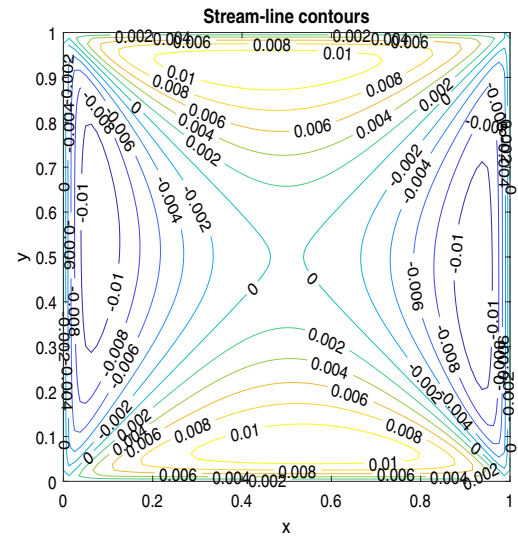

(d) $R e=400$

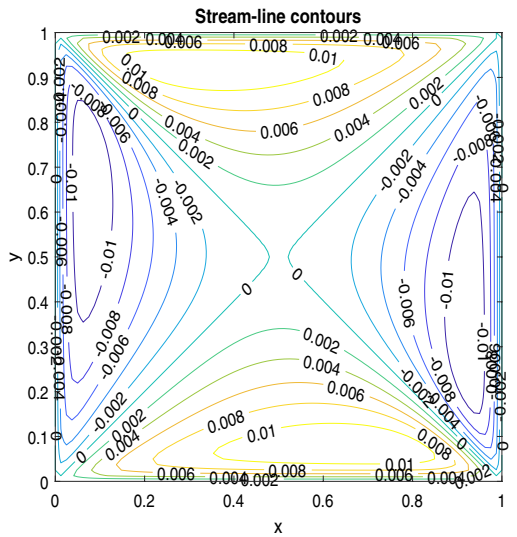

(e) $R e=1000$

Fig. 10 Streamline contours for different values of Reynolds number. a $\operatorname{Re}=10$, b $\operatorname{Re}=50, \mathbf{c} \operatorname{Re}=150$, d $\operatorname{Re}=400$, e $\operatorname{Re}=1000$ with $\operatorname{Pr}=0.7, \mathrm{Da}=10^{-3}, \mathrm{Gr}=10^{3}$, for case 1

is similar to Case 1, except for the location of the center of the primary vortices. The LPV and RPV start changing its position slightly in an upward direction and along the left, and right vertical wall of the cavity respectively with a decrease in Darcy number. Notice that the LPV and RPV are moving in an upward direction for the considered problem while these vortices are shifting in a downward direction in Case 1.

The isotherm contours in the vicinity of the left vertical are oscillating sinusoidal curve, which first decreases then increases and again decreases from the top wall to bottom wall of the cavity, as demonstrated in Fig. 17. The oscillation of these sinusoidal isotherm contours decreases with decreasing of Darcy number from $10^{-1}$ to $10^{-5}$. The isotherm contour with value 0.4 shifts towards the bottom wall of the cavity at $\mathrm{Da}=10^{-3}$ and $\mathrm{Da}=10^{-4}$, as opposed to Case 1.

Figure 18 displays that the velocity profiles in the middle of the cavity decrease and tends to zero with a decrease of Darcy number ranging from $10^{-1}$ to $10^{-5}$. The temperature and averge Nusselt number (see Table 9) for the considered problem are similar to Case 1.

\subsubsection{Effect of Reynolds number}

Figures 19 and 20 respectively present the effect of Reynolds number on streamline and isotherm contours by ranging Re from 10 to 1000 and for fixed $\mathrm{Da}=10^{-3}$ and $\mathrm{Gr}=10^{3}$. The streamline contours behavior is almost similar to the Case 1 of $\operatorname{Re}=10$ for the considered problem at $\mathrm{Re}=50$ and 150 . The streamline contours in the vicinity of the top and bottom wall of the cavity moves in an anticlockwise direction, and is then followed by streamline contours moving in a clockwise direction at $R e=10$. The oscillation of isotherm contours in the vicinity of the left wall increases with an increase of Reynolds number from 10 to 1000 . 


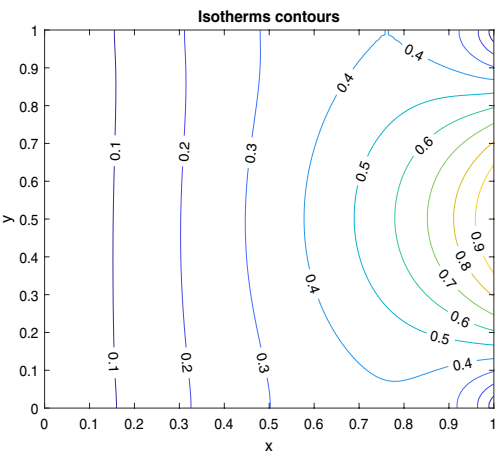

(a) $R e=10$

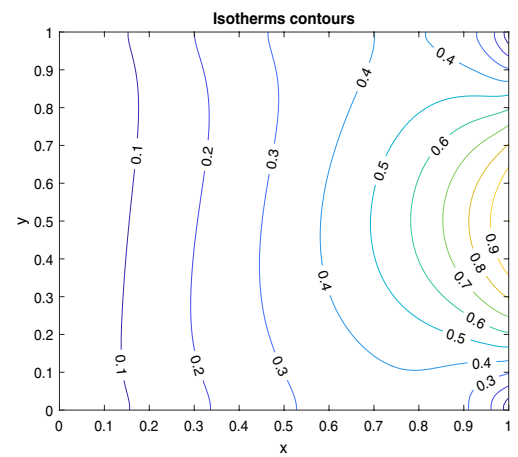

(b) $R e=50$

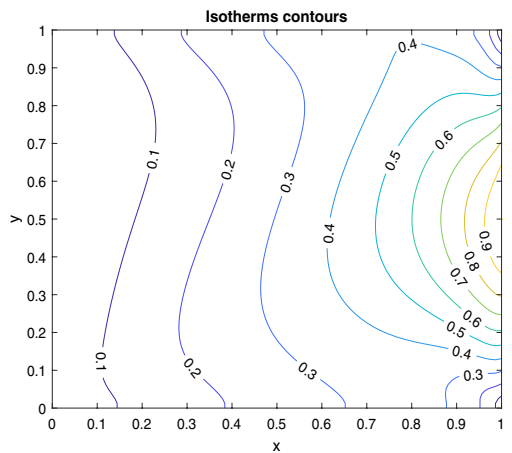

(c) $R e=150$

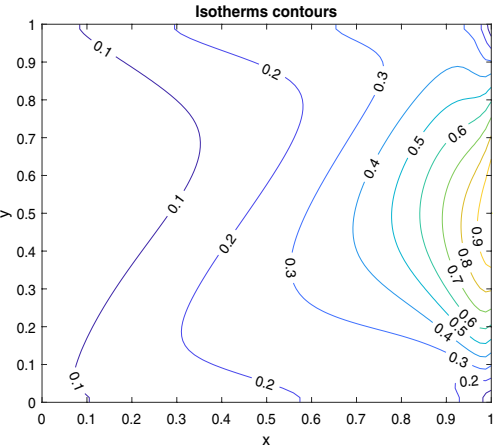

(d) $R e=400$

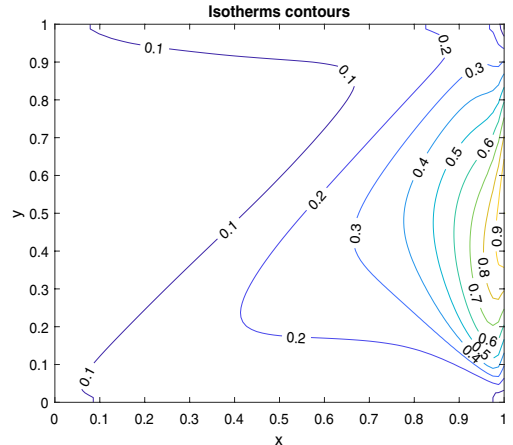

(e) $R e=1000$

Fig. 11 Isotherm contours for different values of Reynolds number. a $\operatorname{Re}=10, \mathbf{b} \operatorname{Re}=50, \mathbf{c} \operatorname{Re}=150, \mathbf{d} \operatorname{Re}=400$, e $\operatorname{Re}=1000$ with $\operatorname{Pr}=0.7, \mathrm{Da}=10^{-3}, \mathrm{Gr}=10^{3}$, for case 1

The effect of Reynolds number on the velocity profiles, temperature, and the average Nusselt number is demonstrated by Fig. 21 for different Reynolds numbers. The velocity profiles in middle of the cavity decreases with an increase of Reynolds number $R e=10-1000$ (see Fig. 21a, b).

Figure $21 \mathrm{c}$ shows that the temperature along the horizontal centerline of the cavity decays with an increase of the Reynolds number. The average Nusselt number along the left vertical wall of the cavity increases with an increase of Reynolds number $\operatorname{Re}=10-1000$, as illustrated in Fig. $21 \mathrm{~d}$.

\section{Conclusions}

The present manuscript describes the mixed convection flow inside a four-sided lid-driven square porous cavity. The top and the bottom walls of the square cavity are adiabatic. The right wall is maintained at a sinusoidal temperature condition while the left wall is at a cold temperature. Depending upon the direction of the moving walls, we have examined two distinct cases. In both cases, the horizontal walls and the vertical walls are moving in the opposite direction with a uniform speed. The numerical results are investigated by ranging the various dimensionless numbers such as Grashof number $\left(10^{3} \leq \mathrm{Gr} \leq 10^{5}\right)$, Darcy number $\left(10^{-5} \leq \mathrm{Da} \leq 10^{-1}\right)$, Reynolds number $(10 \leq R e \leq 1000)$ and keeping the Prandtl number $(\mathrm{Pr}=0.7)$ fixed.

The temperature effect inside the square domain is analyzed with the help of the average Nusselt number. The average Nusselt number along the left wall of the cavity increases with an increase of Grashof number $\left(\mathrm{Gr}=10^{3}\right.$ to $10^{5}$ ), Darcy number $\left(\mathrm{Da}=10^{-5}\right.$ to $10^{-1}$ ) and Reynolds number ( $R e=10$ to 1000 ). The streamline contours and the isotherm contours are analysed for various values of the parameters under consideration. As the temperature on all the four walls is similarly maintained in both the cases, the qualitative nature of the isotherms are also similar in the two cases considered in this study. Due to the change in the direction of the motion of the walls in the two cases, the study reveals that the nature of the streamline contours shift from vertical to the horizontal direction. 


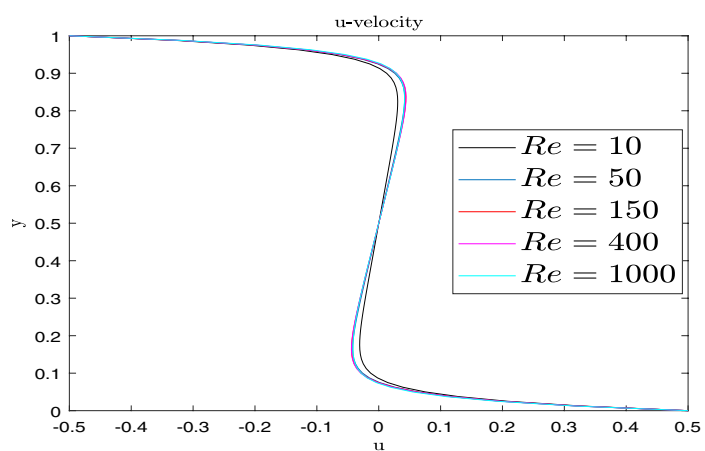

(a) $u$-velocity

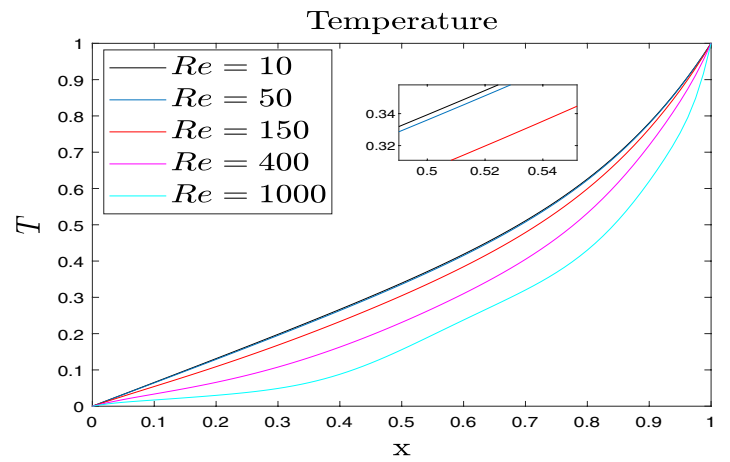

(c) Temperature $(T)$

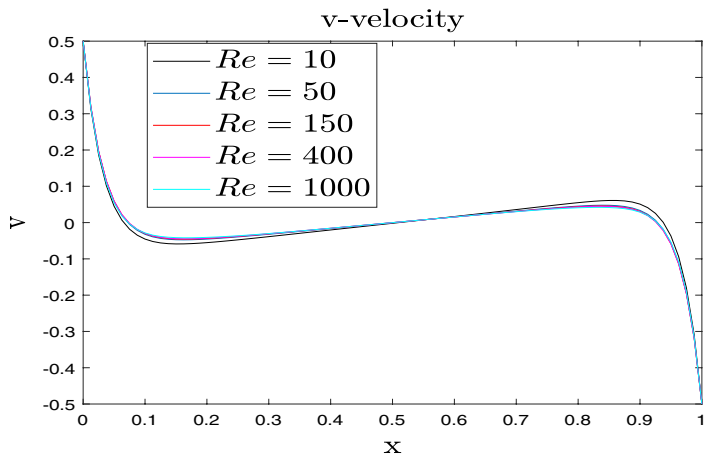

(b) $v$-velocity

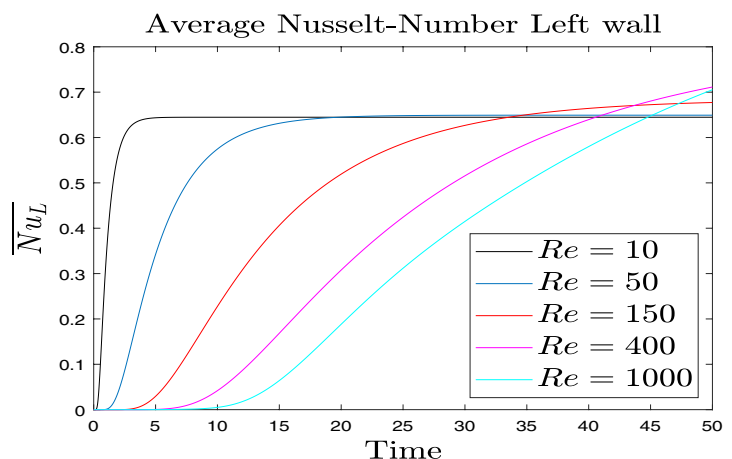

(d) Average Nusselt Number $\left(\overline{N u_{l}}\right)$

Fig. 12 Effect of Reynolds numbers on a $u$-velocity, b $v$-velocity, c temperature $(T)$, d average Nusselt number on left wall with $\operatorname{Pr}=0.7, \mathrm{Da}=10^{-3}, \mathrm{Gr}=10^{3}$, for case 1

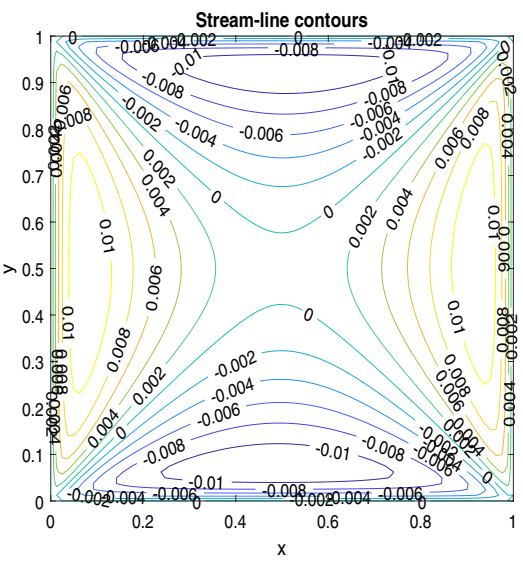

(a) $G r=10^{3}$

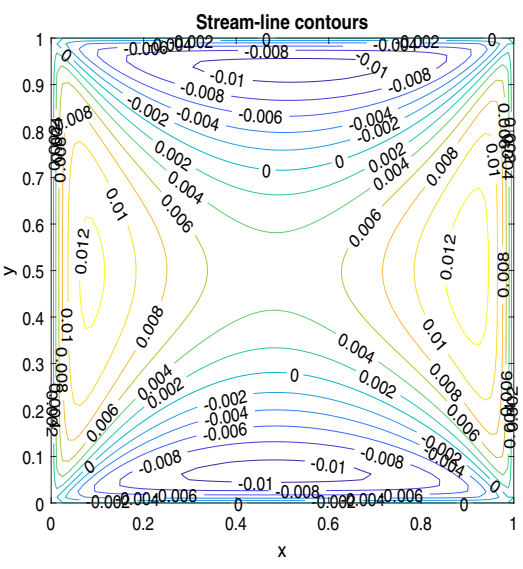

(b) $G r=10^{4}$

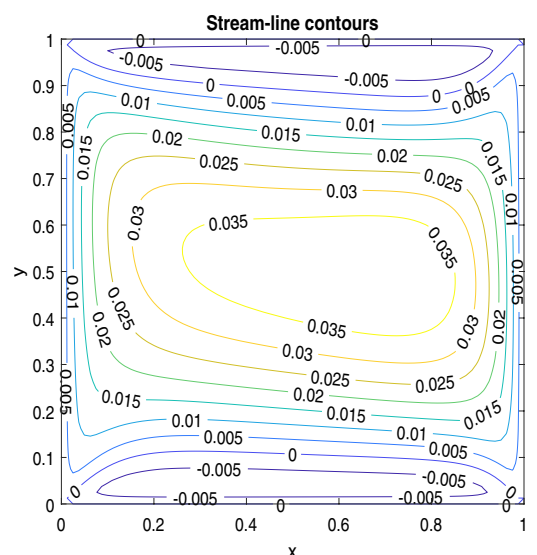

(c) $G r=10^{5}$

Fig. 13 Streamline contours for different values of Grashof number. $\mathbf{a} \mathrm{Gr}=10^{3}, \mathbf{b} \mathrm{Gr}=10^{4}, \mathbf{c} \mathrm{Gr}=10^{5}$ with $\operatorname{Pr}=0.7, \operatorname{Re}=100, \mathrm{Da}=10^{-3}$, for case 2

Results obtained in this study using the stream functionvorticity formulation and ADI method, for particular values of the parameters are found to be in good agreement with the results present in the literature. The problem may be extended by considering the temperature variation on the top and the bottom wall. 


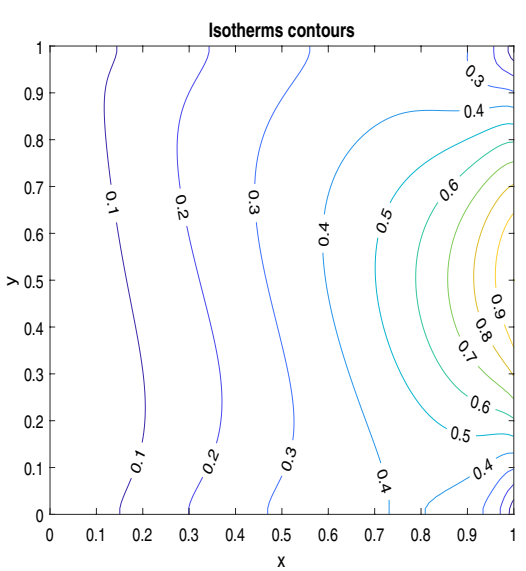

(a) $G r=10^{3}$

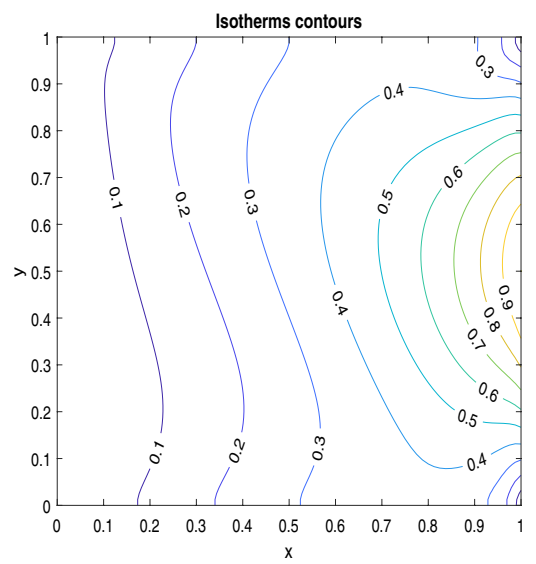

(b) $G r=10^{4}$

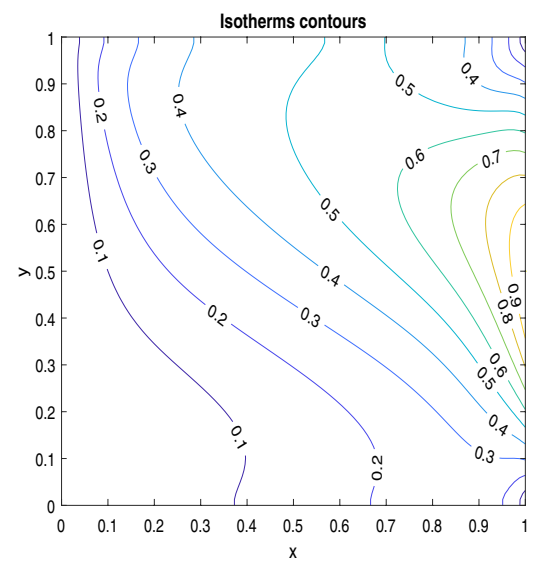

(c) $G r=10^{5}$

Fig. 14 Isotherm contours for different values of Grashof number. $\mathbf{a} \mathrm{Gr}=10^{3}, \mathbf{b ~ G r}=10^{4}, \mathbf{c} \mathrm{Gr}=10^{5}$ with $\operatorname{Pr}=0.7, \operatorname{Re}=100, \mathrm{Da}=10^{-3}$, for case 2

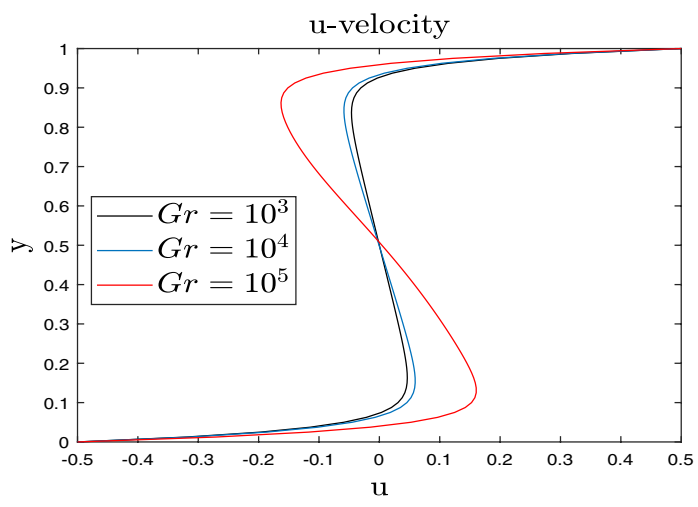

(a) $u$-velocity

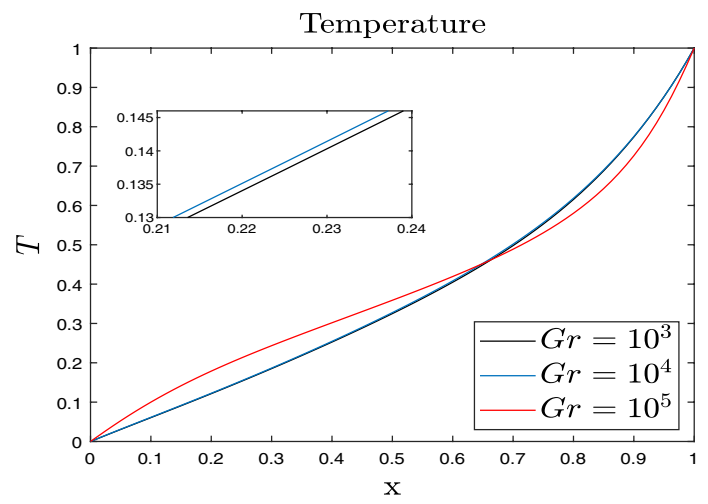

(c) Temperature $(T)$

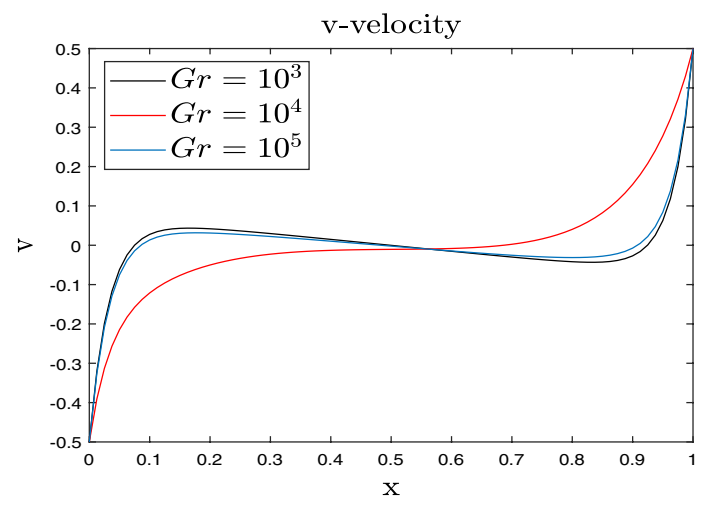

(b) $v$-velocity

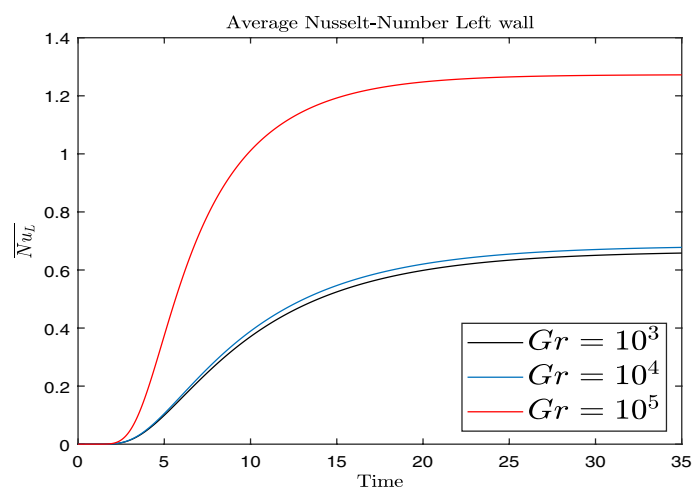

(d) Average Nusselt Number $\left(\overline{N u_{l}}\right)$

Fig. 15 Effect of Grashof numbers on a $u$-velocity, b $v$-velocity, c temperature $(T)$, d average Nusselt number on left wall with $\operatorname{Pr}=0.7, \operatorname{Re}=100, \mathrm{Da}=10^{-3}$, for case 2 
Table 7 Average Nusselt number along the left vertical wall of the cavity at different Grashof numbers for Case 2

\begin{tabular}{lll}
$\overline{\overline{\mathrm{Nu}}}$ & & \\
\hline $\mathrm{Gr}=10^{3}$ & $\mathrm{Gr}=10^{4}$ & $\mathrm{Gr}=10^{5}$ \\
\hline 0.6583 & 0.6778 & 1.272 \\
\hline
\end{tabular}

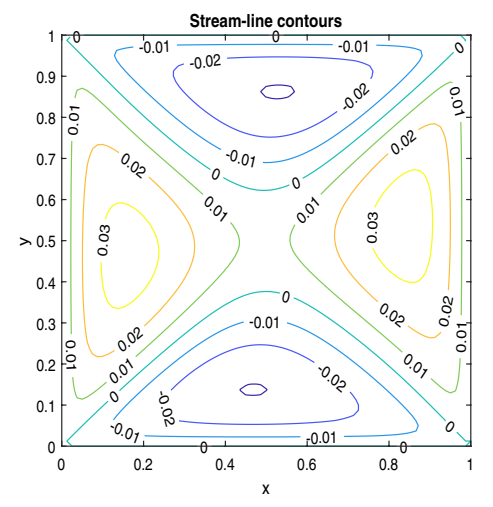

(a) $D a=10^{-1}$

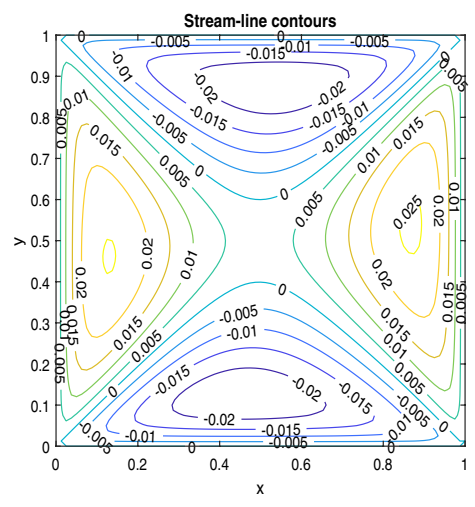

(b) $D a=10^{-2}$

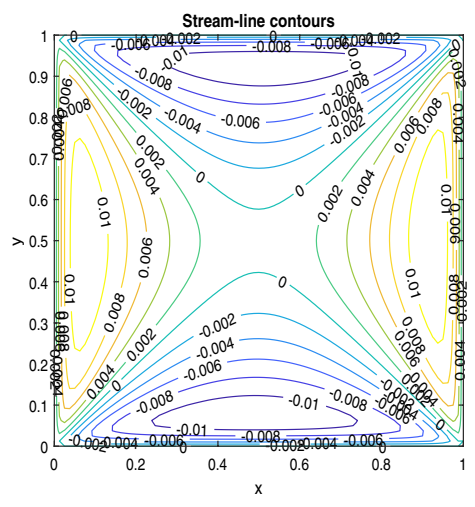

(c) $D a=10^{-3}$

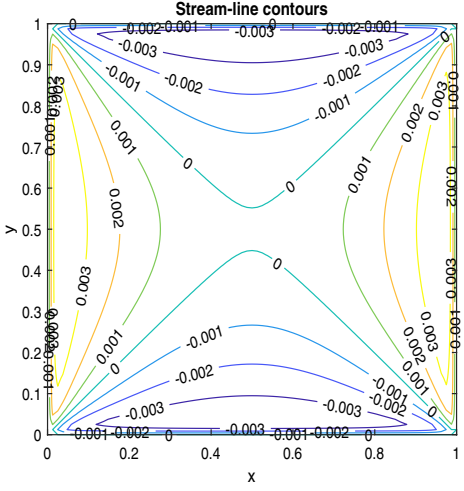

(d) $D a=10^{-4}$

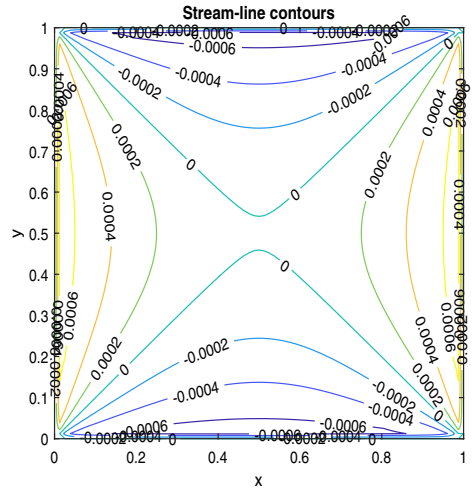

(e) $D a=10^{-5}$

Fig. 16 Streamline contours for $\operatorname{Pr}=0.7, \operatorname{Re}=100, \mathrm{Gr}=10^{3}$ at different values of Darcy number. $\mathbf{a} \mathrm{Da}=10^{-1}, \mathbf{b ~ D a}=10^{-2}, \mathbf{c ~ D a}=10^{-3}, \mathbf{d}$ $\mathrm{Da}=10^{-4}, \mathbf{e D a}=10^{-5}$, for case 2

Table 8 Locations of the centers of the streamline vortices for Case 2 at different Darcy numbers

\begin{tabular}{lllll}
\hline Darcy number & Top primary vortex (TPV) & $\begin{array}{l}\text { Bottom } \\
\text { primary vortex } \\
\text { (BPV) }\end{array}$ & $\begin{array}{l}\text { Left primary vortex (LPV) } \\
\text { Right primary } \\
\text { vortex (RPV) }\end{array}$ \\
\hline $10^{-1}$ & $0.5157,0.8438$ & $0.4688,0.1563$ & $0.1563,0.4532$ & $0.8438,0.5157$ \\
$10^{-2}$ & $0.5313,0.8750$ & $0.4688,0.1250$ & $0.1250,0.4688$ & $0.8750,0.5313$ \\
$10^{-3}$ & $0.5313,0.9375$ & $0.4844,0.0625$ & $0.0781,0.4844$ & $0.9219,0.5313$ \\
$10^{-4}$ & $0.5313,0.9412$ & $0.4844,0.0600$ & $0.0600,0.4844$ & $0.9412,0.5313$ \\
$10^{-5}$ & $0.5400,0.9608$ & $0.5000,0.0212$ & $0.0212,0.5000$ & $0.9608,0.5400$ \\
\hline
\end{tabular}




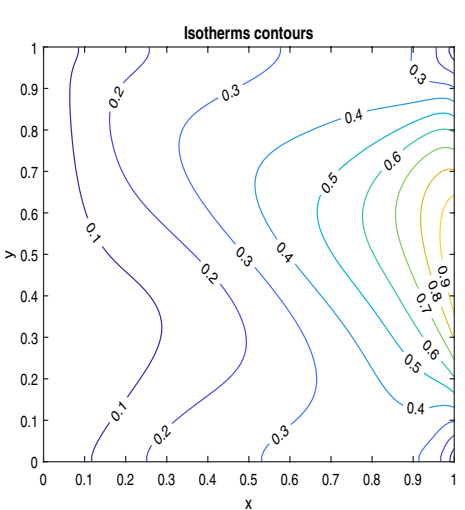

(a) $D a=10^{-1}$

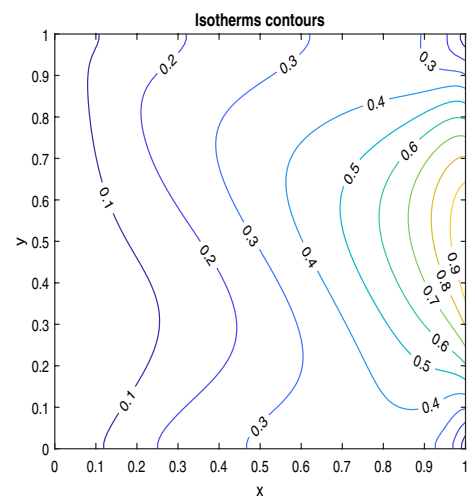

(b) $D a=10^{-2}$

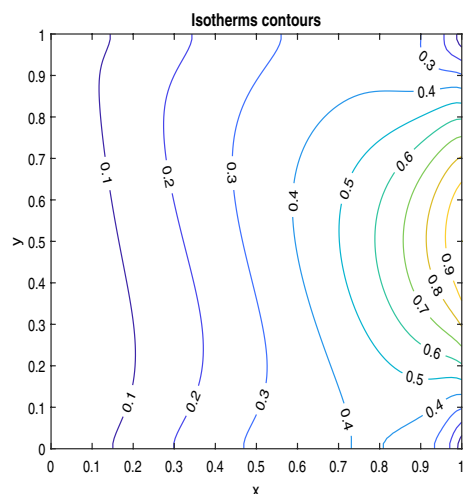

(c) $D a=10^{-3}$

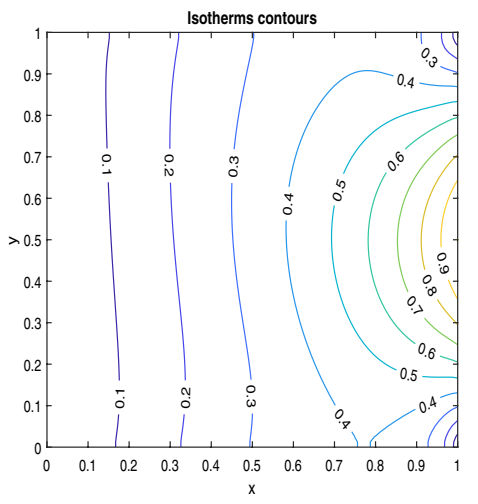

(d) $D a=10^{-4}$

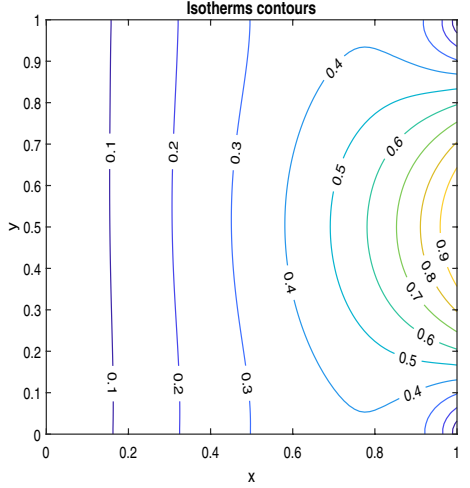

(e) $D a=10^{-5}$

Fig. 17 Isotherm contours for $\operatorname{Pr}=0.7, \operatorname{Re}=100, \mathrm{Gr}=10^{3}$ at different values of Darcy number. $\mathbf{a} \mathrm{Da}=10^{-1}, \mathbf{b ~ D a}=10^{-2}, \mathbf{c ~ D a}=10^{-3}, \mathbf{d}$ $\mathrm{Da}=10^{-4}, \mathbf{e D a}=10^{-5}$, for case 2 


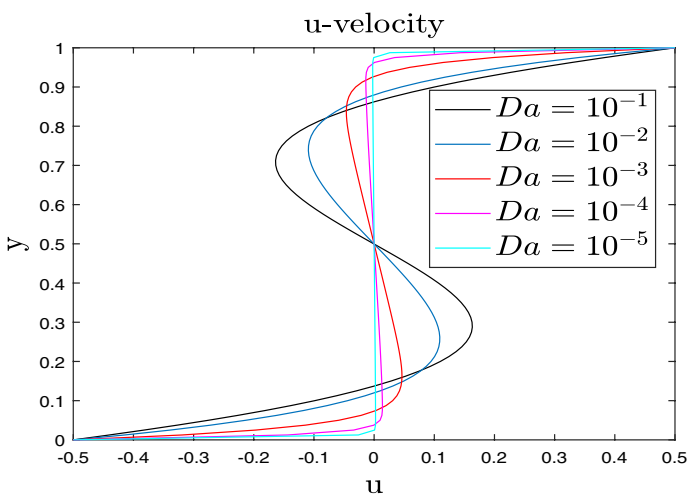

(a) $u$-velocity

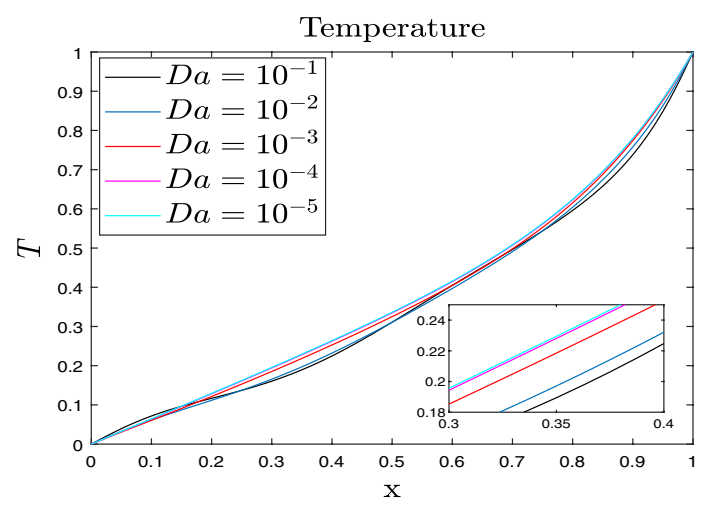

(c) Temperature $(T)$

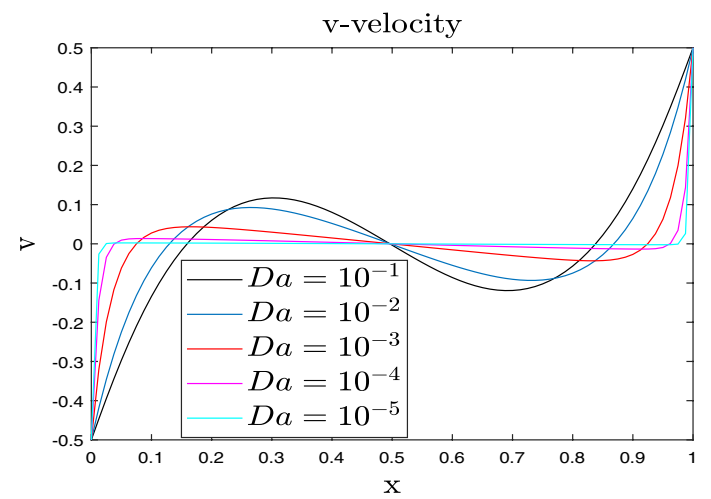

(b) $v$-velocity

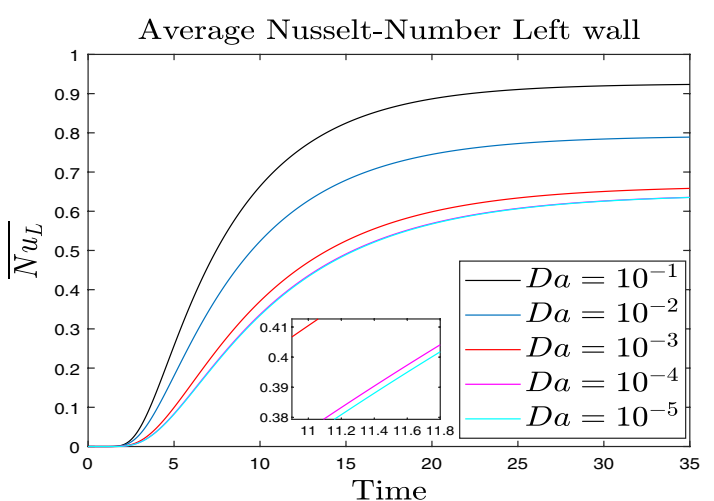

(d) Average Nusselt Number $\left(\overline{N u_{l}}\right)$

Fig. 18 Effect of Darcy numbers on a u-velocity, b $v$-velocity, c temperature $(T)$, d average Nusselt number on left wall with $\operatorname{Pr}=0.7, \operatorname{Re}=100, \mathrm{Gr}=10^{3}$, for case 2

Table 9 Average Nusselt number along the left vertical wall of the cavity at different Darcy numbers for Case 2

\begin{tabular}{lllll}
$\overline{\overline{N u}_{1}}$ & & & \\
\hline $\mathrm{Da}=10^{-1}$ & $\mathrm{Da}=10^{-2}$ & $\mathrm{Da}=10^{-3}$ & $\mathrm{Da}=10^{-4}$ & $\mathrm{Da}=10^{-5}$ \\
\hline 0.9234 & 0.7891 & 0.6583 & 0.6355 & 0.6351 \\
\hline
\end{tabular}




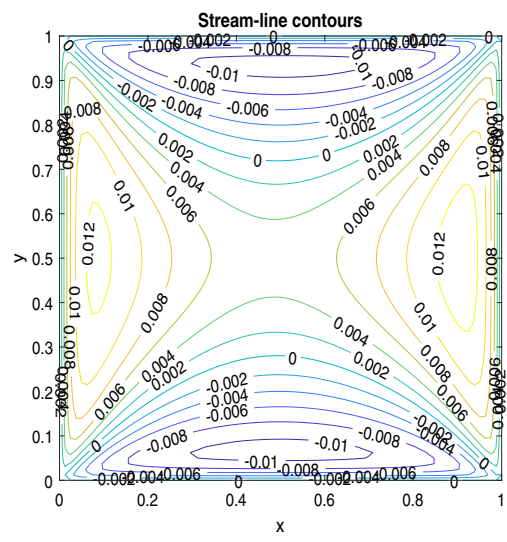

(a) $R e=10$

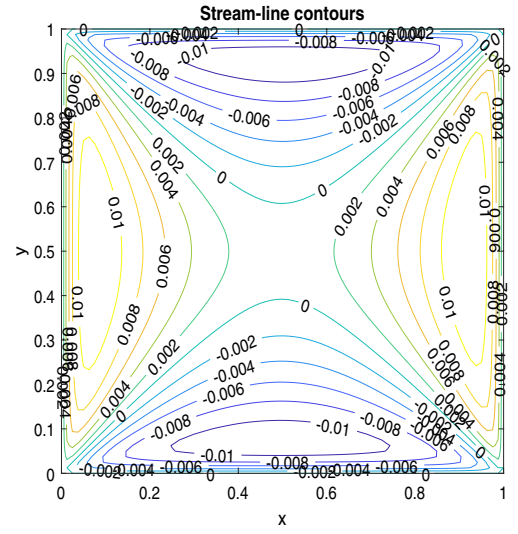

(b) $R e=50$

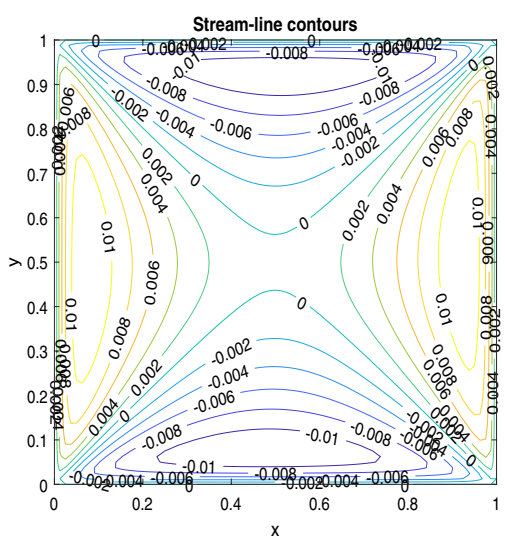

(c) $R e=150$

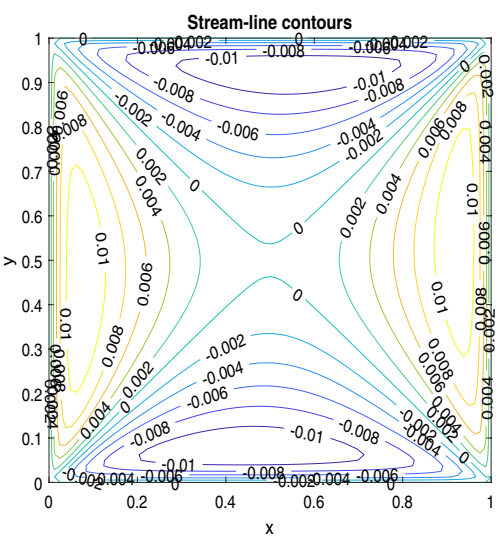

(d) $R e=400$

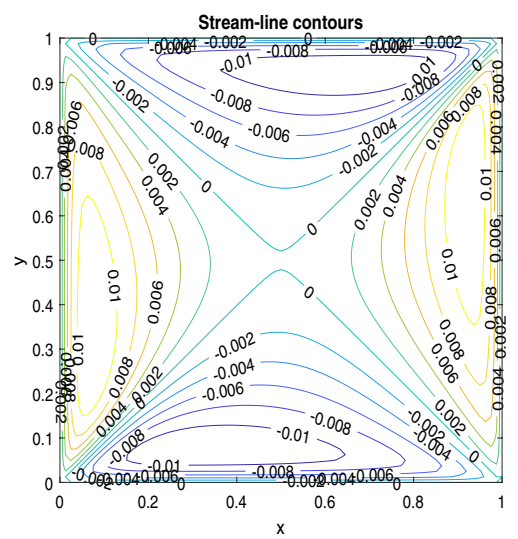

(e) $R e=1000$

Fig. 19 Streamline contours for different values of Reynolds number. a $\operatorname{Re}=10$, b $\operatorname{Re}=50, \mathbf{c} \operatorname{Re}=150, \mathbf{d} \operatorname{Re}=400$, e $\operatorname{Re}=1000$ with $\operatorname{Pr}=0.7, \mathrm{Da}=10^{-3}, \mathrm{Gr}=10^{3}$, for case 2 


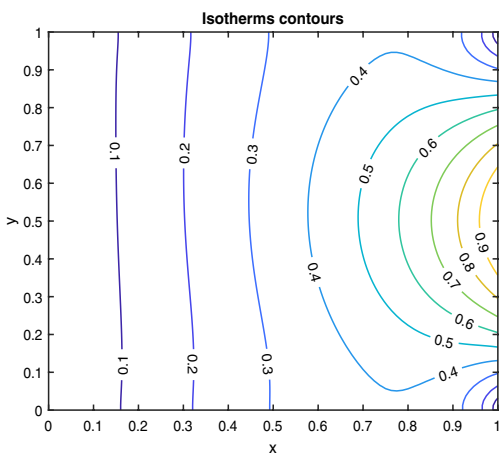

(a) $R e=10$

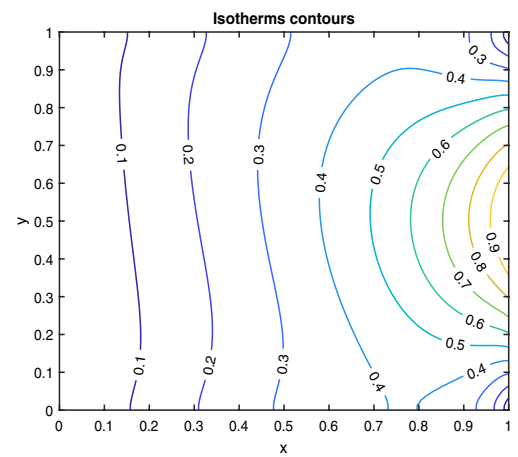

(b) $R e=50$

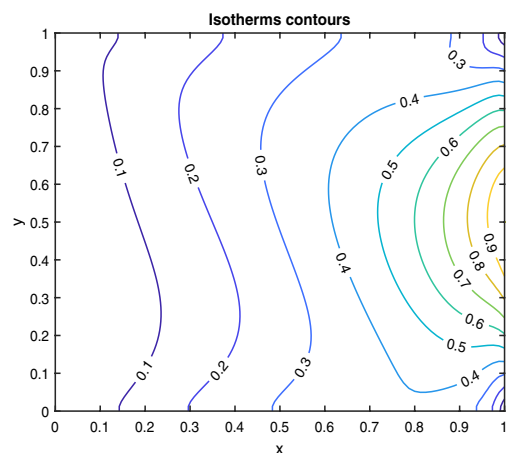

(c) $R e=150$

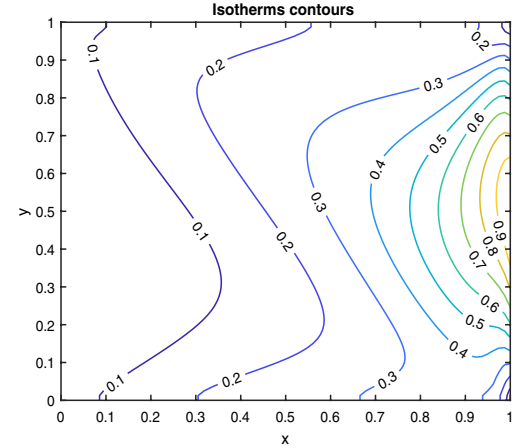

(d) $R e=400$

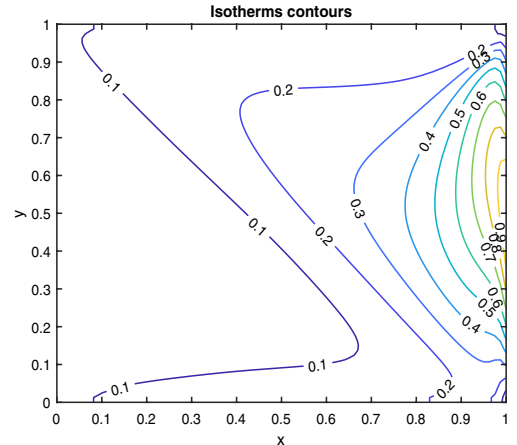

(e) $R e=1000$

Fig. 20 Isotherm contours for different values of Reynolds number. $\mathbf{a} \operatorname{Re}=10, \mathbf{b} \operatorname{Re}=50, \mathbf{c} \operatorname{Re}=150, \mathbf{d} \operatorname{Re}=400$, e $\operatorname{Re}=1000$ with $\operatorname{Pr}=0.7, \mathrm{Da}=10^{-3}, \mathrm{Gr}=10^{3}$, for case 2 


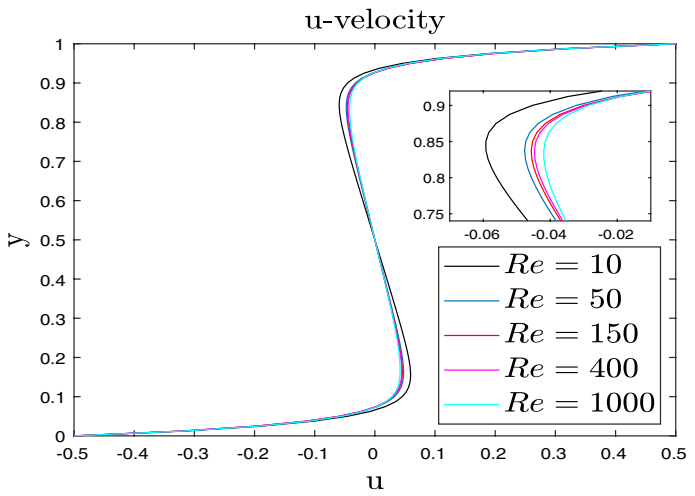

(a) $u$-velocity

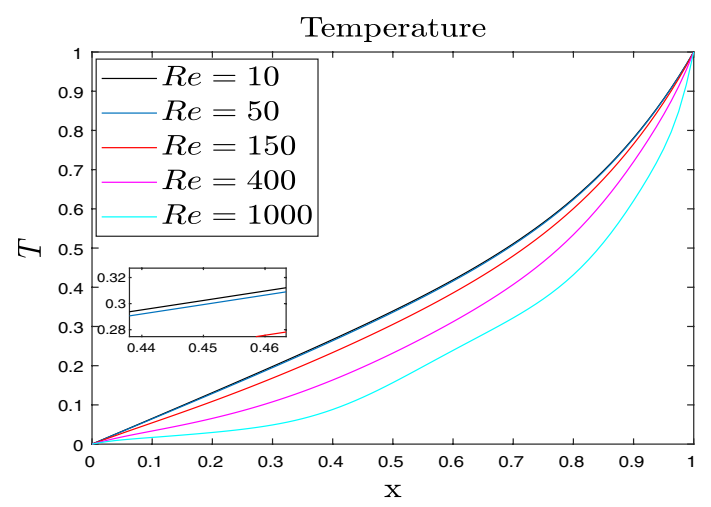

(c) Temperature $(T)$

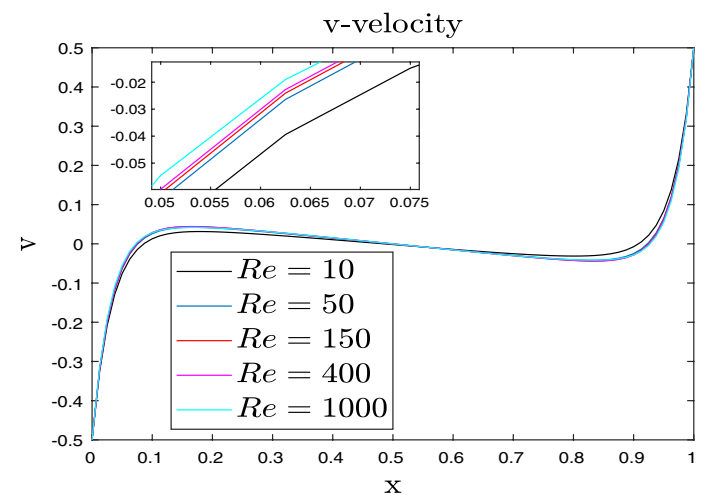

(b) $v$-velocity

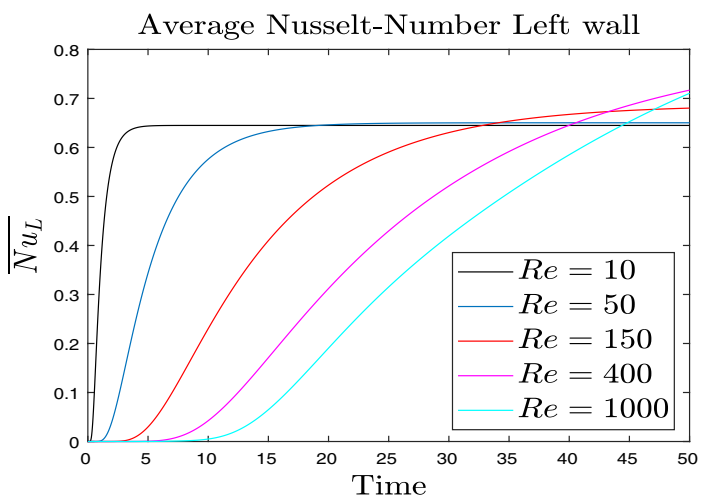

(d) Average Nusselt Number $\left(\overline{N u_{l}}\right)$

Fig. 21 Effect of Reynolds numbers on a u-velocity, b $v$-velocity, c temperature $(T)$, $\mathbf{d}$ average Nusselt number on left wall with $\operatorname{Pr}=0.7, \mathrm{Da}=10^{-3}, \mathrm{Gr}=10^{3}$

Acknowledgements This work is supported by the University Grants Commission, Government ministry of India for providing a research grant for the second author with student id: MAY2018-421012. Furthermore, we would like to thank the referee for his/her valuable suggestions to improve the quality of the manuscript.

Funding This research was funded by the University Grants Commission, Government ministry of India.

\section{Compliance with ethical standards}

Conflict of interest The authors declare that they have no conflict of interest.

\section{References}

1. Ambethkar V, Kumar M (2017) Numerical solutions of 2-d unsteady incompressible flow with heat transfer in a driven square cavity using stream function-vorticity formulation. Int J Heat Technol 35(3):459-473

2. Ambethkar V, Kushawaha D (2017) Numerical simulations of fluid flow and heat transfer in a four-sided lid-driven rectangular domain. arXiv:1705.00707v1 [physics.flu-dyn]
3. Azwadi CSN, Rajab A, Sofianuddin A (2014) Four-sided lid-driven cavity flow using time splitting method of Adams-Bashforth scheme. Int J Autom Mech Eng (IJAME) 9:1501-1510

4. Bagai S, Kumar M, Patel A (2020) The four-sided lid driven square cavity using stream function-vorticity formulation. J Appl Math Comput Mech 19(2):17-30

5. Chapra SC, Canale RP (1989) Numerical methods for engineers. McGraw-Hill companies, New York

6. Chattopadhyay A, Pandit SK, Sarma SS, Pop I (2016) Mixed convection in a double lid-driven sinusoidally heated porous cavity. Int J Heat Mass Transf 93:361-378

7. Chen KT, Tsai CC, Luo WJ (2013) Multiplicity flow solutions in a four-sided lid-driven cavity. Appl Mech Mater 368-370:838-843

8. Karaminejad S, Ketabdari MJ, Boreyri S, Zarech MRB (2013) Simulation of flow in two-and four-sided lid-driven cavity flows. In: The 15th Marine Industries Conference (MIC2013), 29-31-Kish Island

9. Khanafer KM, Chamkha AJ (1998) Mixed convection flow in a liddriven enclosure filled with a fluid-saturated porous medium. Int J Heat Mass Transf 42:2465-2481

10. Kumar DS, Dass AK, Dewan A (2012) Multiple stable solutions for two-and four-sided lid-driven cavity flows using fas multigrid method. Eng e-Trans 7(2):96-106

11. Munshi MJH, Alim MA, Bhuiyan AH, Ali M (2017) Optimization of mixed convection in a lid-driven porous square cavity with internal elliptic shape adiabatic block and linearly heated side walls. 
In: 7th BSME international conference on thermal engineering AIP conference proceedings, vol 1851, pp 020049-1-020049-6

12. Smith GD (1985) Numerical solution of partial differential equations: finite difference methods. Oxford University Press, New York

13. Venkatadri K, Mohiddin SG, Reddy MS (2018) Numerical analysis of unsteady MHD mixed convection flow in a lid-driven square cavity with central heating on left vertical wall. In: Singh MK et al (eds) Applications of fluid dynamics, Lecture Notes in Mechanical Engineering. Springer, Berlin
14. Wahba EM (2009) Multiplicity of states for two-sided and foursided lid driven cavity flows. Comput Fluids 38:247-253

15. Wahba EM (2011) Numerical simulations of flow bifurcations inside a driven cavity. CFD Lett 3:100-110

Publisher's Note Springer Nature remains neutral with regard to jurisdictional claims in published maps and institutional affiliations. 